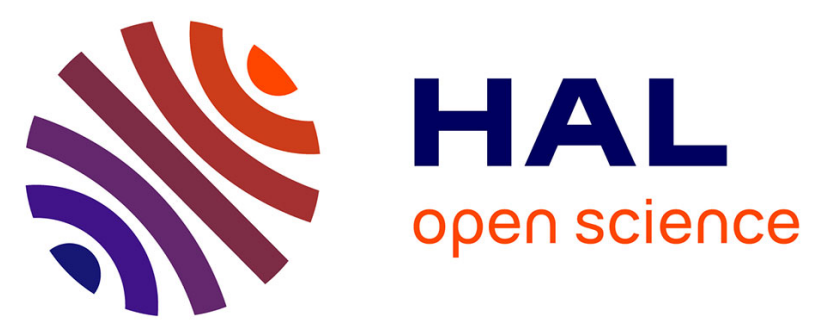

\title{
Pattern and causes of the establishment of the invasive bacterial potato pathogen Dickeya solani and of the maintenance of the resident pathogen $\mathrm{D}$. dianthicola
}

Pauline Blin, Kévin Robic, Slimane Khayi, Jeremy Cigna, Euphrasie Munier, Pauline Dewaegeneire, Angélique Laurent, Yan Jaszczyszyn, Kar-wai Hong, Kok-gan Chan, et al.

\section{To cite this version:}

Pauline Blin, Kévin Robic, Slimane Khayi, Jeremy Cigna, Euphrasie Munier, et al.. Pattern and causes of the establishment of the invasive bacterial potato pathogen Dickeya solani and of the maintenance of the resident pathogen D. dianthicola. Molecular Ecology, 2020, 10.1111/mec.15751 . hal-03028685

\section{HAL Id: hal-03028685 https://hal.science/hal-03028685}

Submitted on 27 Nov 2020

HAL is a multi-disciplinary open access archive for the deposit and dissemination of scientific research documents, whether they are published or not. The documents may come from teaching and research institutions in France or abroad, or from public or private research centers.
L'archive ouverte pluridisciplinaire $\mathbf{H A L}$, est destinée au dépôt et à la diffusion de documents scientifiques de niveau recherche, publiés ou non, émanant des établissements d'enseignement et de recherche français ou étrangers, des laboratoires publics ou privés. 
DR. SLIMANE KHAYI (Orcid ID : 0000-0001-8033-2117)

DR. TATIANA GIRAUD (Orcid ID : 0000-0002-2685-6478)

DR. DENIS FAURE (Orcid ID : 0000-0002-5379-8867)

Article type : Original Article

Pattern and causes of the establishment of the invasive bacterial potato pathogen Dickeya solani and of the maintenance of the resident pathogen $D$. dianthicola

Short title: coexistence of invasive and resident pathogens on potatoes

Pauline Blin ${ }^{1}$, Kévin Robic ${ }^{2,1}$, Slimane Khayi ${ }^{1,3}$, Jérémy Cigna ${ }^{2,1}$, Euphrasie Munier ${ }^{2}$, Pauline Dewaegeneire $^{2}$, Angélique Laurent ${ }^{2}$, Yan Jaszczyszyn ${ }^{1}$, Kar-Wai Hong ${ }^{4}$, Kok-Gan Chan ${ }^{4,5}$, Amélie Beury $^{2}$, Sylvie Reverchon ${ }^{6}$, Tatiana Giraud $^{7}$, Valérie Hélias $^{2}$, Denis Faure ${ }^{1 *}$

1 Paris-Saclay University, CEA, CNRS, Institute for Integrative Biology of the Cell (I2BC), Gifsur-Yvette, France

2 French Federation of Seed Potato Growers (FN3PT/inov3PT), Paris, France

3 Biotechnology Research Unit, National Institute for Agronomic Research (INRA), Rabat, Morocco

4 International Genome Centre, Jiangsu University, Zhenjiang, China

5 Division of Genetics and Molecular Biology, Institute of Biological Sciences, University of Malaya, Kuala Lumpur, Malaysia

6 Univ. Lyon, Université Claude Bernard Lyon 1, INSA-Lyon, CNRS, UMR5240, Microbiologie, Adaptation et Pathogénie (MAP), Villeurbanne, France

7 Ecologie Systématique et Evolution, CNRS, Université Paris-Saclay, AgroParisTech, Orsay, France

This article has been accepted for publication and undergone full peer review but has not been through the copyediting, typesetting, pagination and proofreading process, which may lead to differences between this version and the Version of Record. Please cite this article as doi: $\underline{10.1111 / \mathrm{mec} .15751}$

This article is protected by copyright. All rights reserved 
*Correspondence: denis.faure@cnrs.fr
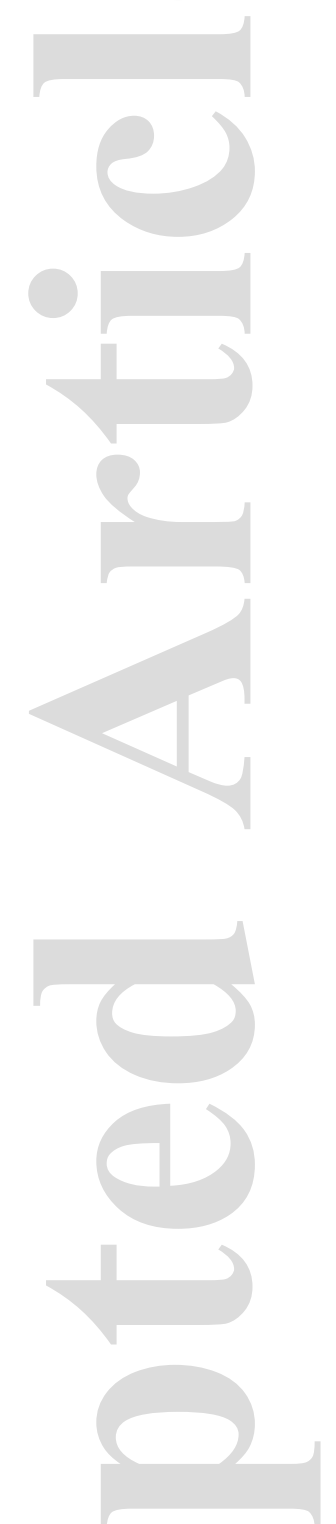

This article is protected by copyright. All rights reserved 


\section{Abstract}

Invasive pathogens can be a threat when they affect human health, food production or ecosystem services, by displacing resident species, and we need to understand the cause of their establishment. We studied the patterns and causes of the establishment of the pathogen Dickeya solani that recently invaded potato agrosystems in Europe by assessing its invasion dynamics and its competitive ability against the closely-related resident $D$. dianthicola species. Epidemiological records over one decade in France revealed the establishment of $D$. solani and the maintenance of the resident $D$. dianthicola in potato fields exhibiting blackleg symptoms. Using experimentations, we showed that $D$. dianthicola caused a higher symptom incidence on aerial parts of potato plants than $D$. solani, while $D$. solani was more aggressive on tubers (i.e. with more severe symptoms). In co-infection assays, $D$. dianthicola outcompeted $D$. solani in aerial parts, while the two species co-existed in tubers. A comparison of $76 \mathrm{D}$. solani genomes (56 of which having been sequenced here) revealed balanced frequencies of two previously uncharacterized alleles, $\mathrm{VfmB}_{\text {Pro }}$ and $V \mathrm{fmB} \mathrm{B}_{\text {Ser, }}$, at the $\mathrm{vfmB}$ virulence gene. Experimental inoculations showed that the $V \mathrm{fmB}_{\text {Ser }}$ population was more aggressive on tubers while the $\mathrm{VfmB}_{\text {Pro }}$ population outcompeted the $\mathrm{VfmB}_{\text {Ser }}$ population in stem lesions, suggesting an important role of the $v f m B$ virulence gene in the ecology of the pathogens. This study thus brings novel insights allowing a better understanding of the pattern and causes of the D.solani invasion into potato production agrosystems, and the reasons why the endemic D.dianthicola nevertheless persisted.

\section{KEYWORDS}

competitive exclusion; Dickeya; invasion; plant pathogens; population genomics; quorumsensing. 


\section{Introduction}

Biological invasions represent major threats to biodiversity as invasive species can replace native species. Evolutionary and ecological studies are of major importance for understanding the patterns and causes of biological invasions and plant and animal invasions have been extensively studied since the $19^{\text {th }}$ century (Burns, Murphy, \& Zheng, 2018; Cadotte, Campbell, Li, Sodhi, \& Mandrak, 2018; MacDougall, Gilbert, \& Levine, 2009). Evolutionary and ecological approaches aim at elucidating the dynamics of invasions as well as the advantages of invaders that allow them to outcompete resident species (Facon et al., 2006; Gladieux et al., 2015). Although microbial invasions can represent important threats to ecosystems and human societies, they have been much less studied than invasive plants and animals (Gladieux et al., 2015; Kinnunen et al., 2016; Litchman, 2010; Mallon, Elsas, \& Salles, 2015; Perkins, Leger, \& Nowak, 2011).

In this work, we used evolutionary and ecological approaches for understanding the patterns and causes of the establishment of the bacterial pathogen Dickeya solani in potato agrosystems and the maintenance of the resident, related competitor $D$. dianthicola. There are two major clades of potato bacterial pathogens in Europe, Pectobacterium and Dickeya, two sister genera among the order Enterobacterales (Gamma-proteobacteria). While some Pectobacterium species had settled since before the 1970s in potato agrosystems in Europe (Pérombelon, 2002), Dickeya species invaded later, in two successive waves. Mostly isolated from ornamental plants such as Dianthus, Dahlia and Begonia, D. dianthicola has been reported in Europe as early as the 1950s. Isolation of $D$. dianthicola from Solanum tuberosum dates back to the 70's (Toth et al., 2011; Parkinson et al., 2009). Nowadays, D. dianthicola is considered an endemic pathogen of potato plants in Europe. It is present in all continents, including North-America, where it was associated with disease outbreaks in potato farms in 2015 (Oulghazi et al., 2017; Patel, Baldwin, Patel, Kobayashi, \& Wyenandt, 2019; Sarfraz et al., 2018; Toth et al., 2011; Wright et al., 2018).

Dickeya solani has emerged in potato production systems in Europe much more recently, in the early 2000s (Toth et al., 2011; van der Wolf et al., 2014). Aside from potatoes, $D$. solani has been isolated from ornamental plants such as Hyacinthus, Iris and Muscari, but the primary host(s) of that species remain(s) uncertain (Chen, Zhang, \& Chen, 2015; Slawiak et al., 2009; van der Wolf et al., 2014). Population genomics revealed little genetic variation in $D$. solani, in agreement with a recent spread of this species with a bottleneck associated to the introduction and/or invasion (Golanowska et al., 2018; Khayi et al., 2015). An analysis of 20 D. solani genomes identified a non-synonymous single nucleotide polymorphism (SNP) in each of the two fliC and fliN genes that are involved in bacterial motility (Khayi et al., 2015). Horizontal gene transfer events from $D$. dianthicola to $D$. solani have been documented, adding or replacing genomic fragments (Khayi et al., 2015).

This article is protected by copyright. All rights reserved 
The $D$. solani invader, as well as the $D$. dianthicola and Pectobacterium spp. residents, are necrotrophic pathogens causing similar symptoms, resulting in blackleg disease on stems and soft-rot disease of tubers (Charkowski 2018; Toth et al., 2011). All these bacteria secrete plant cell-wall macerating enzymes (including the pectate lyases) and proliferate by assimilating the plant cell remains, including iron by producing the chrysobactin and achromobactin siderophores. Pectate lyases, i.e., PelA, PelD and especially PelE, play a key role in the initiation of the plant cell wall maceration. Using pectin as a substrate, these enzymes release oligosaccharides that elicit expression of other plant-cell wall macerating enzymes in a positive feedback loop (Duprey, Nasser, Léonard, Brochier-Armanet, \& Reverchon, 2016). Noticeably, the pelA gene is truncated, hence non-functional, in D. dianthicola (Duprey et al., 2016; Raoul des Essarts et al., 2019). The secretion of these virulence factors is a costly process that is tightly regulated, being induced by the perception of chemicals that inform the pathogen on plant host physiology (mainly plant sugars) and on the size of its own population (quorum-sensing signals) (Leonard, Hommais, Nasser, \& Reverchon, 2017). Dickeya pathogens synthetize and sense two types of quorumsensing signals, the $\mathrm{N}$-acylhomoserine lactones and $\mathrm{Vfm}$ compounds, which activate virulence gene expression when a threshold concentration of signals (reflecting the number of cells in the environment) is reached (Crépin et al., 2012; Nasser et al., 2013; Potrykus, Golanowska, Hugouvieux-Cotte-Pattat, \& Lojkowska, 2014; Potrykus, , Hugouvieux-Cotte-Pattat, \& Lojkowska, 2018). By modulating the synthesis of virulence factors, the quorum-sensing signals participate in the transition from an oligotroph lifestyle in soils and surface waters, where Dickeya and Pectobacterium bacteria are rare, to a copiotroph lifestyle on a plant host, where they increase in number by several orders of magnitude (Laurila et al., 2008; Potrykus et al., 2016). Functional differences such as metabolic capacities have been identified between $D$. solani and $D$. dianthicola by comparative genomics, transcriptomics and biochemical approaches (BellienyRabelo et al., 2019; Raoul des Essarts et al., 2019), but fitness differences within host plants remain only partially investigated (Czajkowski et al., 2013; Czajkowski, de Boer, Velvis, \& van der Wolf, 2010; Raoul des Essarts et al., 2019; Shyntum et al., 2019). Whether the potato host could be differentially exploited by $D$. dianthicola and $D$. solani and whether the two pathogens stably coexist by using different ecological niches or compete directly when exploiting this host remain unsolved questions, despite their importance for understanding the $D$. solani invasion.

Here, we combined epidemiological records in potato fields, experimental inoculations and population genomics to contribute to our understanding of the patterns and ecological determinants of $D$. solani invasion. Epidemiological records over one decade in France allowed to compare the relative abundances of the $D$. solani invader, the $D$. dianthicola resident and Pectobacterium spp. Using experimental populations and plant assays in greenhouse, with a 
diversity of strains and of ecological conditions, we then focused on $D$. solani and $D$. dianthicola to determine the ecological traits that could facilitate or constrain $D$. solani establishment, including competition or ecological niche differences between the two Dickeya species. Finally, we obtained the genomes of $56 \mathrm{D}$. solani isolates that we analyzed together with 20 genomes previously published (Khayi et al., 2015), revealing novel variations in D. solani population. We focused on the quorum-sensing gene $\operatorname{vfm} B$, because it is known to be involved in virulence, to investigate whether its variation, which has not been studied so far, could be associated to invasion traits in $D$. solani.

\section{Material and Methods}

\subsection{Pathogen sampling and identification in potato fields with blackleg disease symptoms}

Pectinolytic bacteria were isolated from symptomatic plant tissues on crystal violet pectate medium (Hélias, Hamon, Huchet, Wolf, \& Andrivon, 2012). Isolates were purified on agar plates and characterized at the genus (Dickeya and Pectobacterium) and species ( $D$. solani and $D$. dianthicola) levels using the PCR primers listed in Table S1. Two sampling strategies were used. From 2004 to 2015, a national inventory was conducted in French potato producing areas. Each year (with the exception of the year 2006), fields exhibiting blackleg symptoms were randomly sampled by collecting diseased plants from which isolates were recovered and characterized. From 541 fields, around 1600 isolates were collected and taxonomically characterized. In parallel, from 2013 to 2016, 19 of the potato fields exhibiting more than $1 \%$ of blackleg incidence were deeply sampled. Around 30 plants with blackleg symptoms were collected along a transect in each field, and a single isolate was retained from each of the 548 plants. The resulting 548 isolates were characterized taxonomically.

\subsection{Dickeya solani and $D$. dianthicola strains used in plant assays}

Five D. dianthicola strains (RNS11-47-1-1A, CFBP1888, CFBP2982, CFBP2015, MIE34) and five D. solani strains (3337 = RNS08-23-3-1A, IPO2222, RNS05-1-2A, Ds0432.1, PPO9019) were used to produce plant inoculation assays. The strains were chosen to be representative of the diversity of the populations, i.e. from different geographical locations, isolation years or host of collection (potato plants vs ornamentals), and regarding phylogeny placement with neutral markers (Figure S1 and see characteristics and genome accession numbers in Table S2). Phylogeny analyses were conducted using MEGA7 (Kumar, Stecher, \& Tamura, 2016). Bacterial strains were stored at $-80^{\circ} \mathrm{C}$ in glycerol $(25 \% \mathrm{vol} / \mathrm{vol})$. They were cultivated in TY medium (tryptone $5 \mathrm{~g} \cdot \mathrm{L}^{-1}$; yeast extract $\left.3 \mathrm{~g} \cdot \mathrm{L}^{-1}\right)$ with or without agar $\left(15 \mathrm{~g} \cdot \mathrm{L}^{-1}\right)$ at $28^{\circ} \mathrm{C}$.

This article is protected by copyright. All rights reserved 


\subsection{Plant and tuber inoculations by $D$. solani and $D$. dianthicola strains and experimental populations}

In our plant and tuber assays, we use the term virulence as the capacity of the pathogen to parasitize a host (symptom incidence) and the term aggressiveness as the degree of damage caused by a pathogen on a host (symptom or disease severity). The terms used to describe pathogens behaviors (virulence, symptom incidence, aggressiveness, disease severity index, fitness and competitive index) are defined more extensively in Supplementary Methods 1 (SM1). We designed the whole plant experiments based on our knowledge of the pathosystem, to investigate contrasting conditions relevant for the disease expression in fields. Each bacterial strain was cultivated individually before being used for plant and tuber inoculations, either separately or assembled in populations. To constitute experimental populations of $D$. solani, $D$. dianthicola, or mixtures of the two species, cell suspensions of different strains were assembled just before plant inoculation. Potato plants (S. tuberosum var. Bintje) and hyacinths (Hyacinthus orientalis var. Delft blue) were cultivated in horticultural compost in individual pots $(2 \mathrm{~L})$ in a greenhouse at around $23^{\circ} \mathrm{C}$ (minimum $20^{\circ} \mathrm{C}$ maximum $28^{\circ} \mathrm{C}$ ) with a 12-hour photoperiod. For mimicking plant infection by soil pathogens, potato plants (three weeks post-tuber plantation) and hyacinths (four weeks post-bulb plantation) were inoculated by watering the substrate with a pathogen cell suspension at $10^{9}$ colony-forming units (CFU) per pot. This inoculation load was calibrated to obtain a large range (20 to $60 \%$ ) of symptom incidence in the unwounded potato plant condition (Raoul des Essarts et al., 2015). In a wounded condition, the potato roots were wounded with a sterile knife before infection. The symptom monitoring was adapted from Raoul des Essarts et al. (2015). The number of symptomatic potato plants (blackleg disease) and hyacinths (soft-rot disease) was recorded twice a week (see examples of symptoms in Figure 1ab). For each strain and experimental population and unwounded and wounded conditions, the number of symptomatic and asymptomatic plants (hence two symptom classes that were used in statistical comparisons) was counted at 70 days post infection (dpi) using 15 potato plants (up to 25 potato plants when indicated) and 8 hyacinths. A set of non-inoculated plants was used as an asymptomatic control. In order to facilitate visualization of the virulence data in the figures, they are presented as a percentage of symptomatic plants (symptom incidence).

In tuber assays, potato tubers ( $S$. tuberosum var Bintje) were inoculated using a tip to inject $10 \mu \mathrm{L}$ of calibrated pathogen suspensions. Based on previous results (Raoul des Essarts et al., 2019), we chose two different inoculum loads of strains and experimental populations (10 $\mathrm{CFU}$ and $10^{5}$ CFU per tuber) to obtain a large range of symptom severity, to compare the capacity to initiate maceration at different population sizes, to investigate fitness of the two pathogens in two contrasting ecological conditions. After 5 days of incubation at $24^{\circ} \mathrm{C}$, the tubers were cut in half

This article is protected by copyright. All rights reserved 
and scored based on five aggressiveness classes according to the symptom severity in each tuber (from no symptom to the most severe symptoms; Figure 1c). For each strain and experimental population, at least 10 tubers were inoculated. A set of non-inoculated tubers was used as an asymptomatic control. In order to facilitate the presentation of the aggressiveness data in the figures, results are presented as normalized disease severity index (DSI) of which values, ranging from 0 to 100 (arbitrary unit), increase with symptom severity. DSI was calculated using the five aggressiveness classes assigned to 10 tubers, see formula in Supplementary

\section{Methods 1 (SM1).}

Emerging lesions from potato stems and hyacinths and rotting tissue recovered from tubers at 5 dpi were collected and frozen at $-80^{\circ} \mathrm{C}$, and then used to quantify the abundance $D$. solani and $D$. dianthicola pathogens within hosts by Taq Man qPCR as described in Supplementary Methods 2 (SM2). Assessing $D$. dianthicola and $D$. solani in inoculum and plant tissues (potato stems and tubers, and hyacinths) permitted the calculation of competitive index $(\mathrm{Cl})$ values following the formula presented in SM1. A Cl value equal to one indicated an equal fitness between $D$. solani and $D$. dianthicola, $\mathrm{Cl}$ value greater than one indicated a fitness advantage to $D$. solani, and $\mathrm{Cl}$ value below one indicated a fitness advantage to $D$. dianthicola.

\subsection{Dickeya solani population genomics}

Total DNA was purified from each of $56 \mathrm{D}$. solani isolates (their characteristics in Figure S2) using MasterPure complete DNA and RNA purification kit (Epicentre, Illumina). After quantity and quality controls of extracted DNA using a NanoDrop (ND 1000) device and agarose gel electrophoresis at $1.0 \%(\mathrm{w} / \mathrm{v})$, paired-end libraries were constructed for each strain and then sequenced using High Output Sequencing kit with $75 \times 2$ cycles on and Illumina NextSeq500 sequencer (CNRS, Gif-sur-Yvette, France). For each strain, 2.4 to 18 million reads were obtained, corresponding to an average coverage ranging from $37 \times$ to $270 \times$. Paired-end reads were trimmed (quality score threshold 0.05 ) and mapped against the $D$. solani reference genome, from the 3337 strain, at mild stringency threshold ( $80 \%$ identity on $50 \%$ read length) using CLC Genomics Workbench version 10.1.3 (Khayi et al. 2015). The mapping was used for the detection of SNPs and InDels using the variant calling tool from CLC genomic workbench version 10.1.3. Only SNPs and InDels ( 1 to 9 nucleotides) with a high occurrence ( $\geq 99 \%$ of the reads) in the mapping step were retained. The representation of allelic variants in $D$. solani population was performed using PHYLOViZ (Francisco et al., 2012). 


\subsection{Characterization of the fitness differences between Dickeya solani strains carrying the}

\section{$\mathrm{VfmB}_{\text {Ser }}$ and $\mathrm{Vfm} \mathrm{B}_{\text {Pro }}$ alleles}

The structure of the VfmB proteins was modeled and represented using the Phyr2 and EzMol web portals (Kelley, Mezulis, Yates, Wass, \& Sternberg, 2015; Reynolds, Islam, Sternberg, 2018). Given the known role in virulence of the vfm gene cluster (Nasser et al., 2013), we investigated the impact on virulence of a SNP detected in the vfm gene cluster : we assessed virulence differences between $D$. solani strains exhibiting either a serine (allele $\mathrm{VfmB}_{\mathrm{Ser}}$ ) or a proline (allele $\mathrm{VfmB} \mathrm{B}_{\mathrm{Pro}}$ ) at the position 55 of the $\mathrm{VfmB}$ protein. Using genomic data, we chose four isolates carrying $\mathrm{VfmB}_{\text {Pro }}$ (IPO2222, MIE35, AM3a and 3337) and four isolates carrying $\mathrm{VfmB}_{\text {Ser }}$ (Ds0432.1, RNS10-27-2A, Sp1a and M21a), the four isolates in each group differing at other positions in genomes (genome accession numbers and characteristics in Tables S3 and S4). Plant inoculation assays (on potato tubers and stems) were performed following the same protocols as described above using pure strains and mixtures as inocula. In the figures, virulence was presented as the percentage of symptomatic plants (in potato stem infections assay) and aggressiveness by DSI values (in potato tuber infection assays).

To measure their relative fitness, co-inoculation assays were also performed with $\mathrm{VfmB}_{\mathrm{Ser}}$ and $\mathrm{VfmB}_{\text {Pro }}$ experimental populations, and their relative abundance was quantified by shot gun sequencing of DNA extracted from inoculum and from lesions. From 12 to 28 million reads were obtained for each sample, corresponding to an average coverage of $D$. solani genomes ranging from $180 \times$ to $420 \times$. Sequencing $(75 \times 2$ cycles) was performed using an Illumina NextSeq500 at the I2BC platform (CNRS, Gif-sur-Yvette, France) and Illumina MiSeq platform (University of Malaya, Kuala Lumpur, Malaysia). The trimmed reads were mapped on $D$. solani vfmB gene for quantifying the relative abundance of the two $\mathrm{VfmB}_{\text {Ser }}$ and $\mathrm{VfmB}_{\text {Pro }}$ alleles in each sample using the CLC genomic workbench version 10.1.3. The relative abundances of the alleles permitted calculation of $\mathrm{Cl}$ values of the $\mathrm{VfmB}_{\text {Ser }}$ and $\mathrm{VfmB}_{\text {Pro }}$ populations (see SM1). A Cl value equal to one indicated an equal fitness between $D$. solani $V \mathrm{fmB}_{\text {pro }}$ and $\mathrm{VfmB} \mathrm{B}_{\text {Ser }}$ populations, $\mathrm{Cl}$ value greater than one indicated a fitness advantage to $\mathrm{VfmB} \mathrm{B}_{\text {Pro }}$, and $\mathrm{Cl}$ value less than one indicated a fitness advantage to $\mathrm{VfmB}_{\text {ser }}$. Finally, we used transcriptomics to identity the genes that were differentially expressed between IPO2222 ( $\left.\mathrm{VfmB}_{\text {Pro }}\right)$ and Ds0432.1 (VfmB $\mathrm{Ber}_{\text {Ser }}$ D. solani strains when they grew inside potato tubers (SM2). Transcriptomes were compared as described by Raoul des Essarts et al. (2019).

\subsection{Statistical analyses}

The slopes of the dynamics of the percentages of Dickeya-, Pectobacterium-, D. solani- and D. dianthicola-positive fields were analyzed using F-tests and ANCOVAs using GraphPad Prism 
version 8.4.2 for MacOS, GraphPad Software (www.graphpad.com). A Chi-squared method was used to test the non-random distribution of taxa in each field.

In all the plant assays, the non-inoculated plants and tubers remained asymptomatic, and this control condition was not considered in statistical analyses. The two symptom levels (with or without symptoms) of potato plants and hyacinths virulence assays, the five symptom classes in tuber aggressiveness assays, and gene expression levels were compared among treatments (the different pathogens) by Kruskal-Wallis tests $(\alpha=0.05)$ because the data significantly deviated from normality. Then, post-hoc Tukey tests were run to assess what pairwise differences were significant. When the bacterial strains were inoculated separately on plants and tubers, we compared the $D$. solani and $D$. dianthicola species traits using each bacterial strain as a replicate of the considered species. When the bacterial strains were inoculated altogether to constitute an experimental population, we compared the $D$. solani and $D$. dianthicola species traits using at least two independent replicates of the experiments.

In competition assays, the hypothesis of balanced abundances of the two competitors was tested by comparing the calculated $\mathrm{Cl}$ values to one using a Kruskal-Wallis test. Analyses were carried out with the software R (http://www.R-project.org) using the packages RcmdR and nparcomp.

\section{Results}

\subsection{Little co-occurrence of $D$. dianthicola and $D$. solani in potato fields}

A monitoring of the Pectobacterium and Dickeya populations was performed over one decade (2004-2015) in France. Each year, we recorded the number of fields from which we isolated each taxon (Table S5). These data informed on the dynamics of the different pathogen populations. Over the period, Dickeya was detected in $10 \%$ to $35 \%$ of the sampled fields (Figure 2a). A moderate increase of the percentage of Dickeya-positive fields was observed over time $(F=5.05$; $\mathrm{DFn}=1 ; \mathrm{DFd}=9 ; \mathrm{p}=0.05 ; \mathrm{R}^{2}=0.36$ ). Although decreasing in incidence, the resident Pectobacterium species remained the most widespread $(67 \%$ in the most recent sampling year 2015). As expected, the slopes of the dynamics of the percentage of Dickeya- and Pectobacterium-positive fields diverged $\left(F=10.11\right.$; $\left.D F n=1 ; D F d=18 ; p=5 \times 10^{-3}\right)$. Among the Dickeya isolates, only two species were identified, $D$. dianthicola and $D$. solani (Figure 2b). The slopes of $D$. dianthicola-positive $(F=1.35 ; D F n=1 ; D F d=9 ; p=0.28)$ and $D$. solani-positive $(F=$ 3.10; $D F n=1 ; D F d=9 ; p=0.11$ ) field percentages were not different from base line and were not different from each other $(F=0.26 ; D F n=1 ; D F d=18 ; p=0.62)$. This suggests that the $D$. solani invasion did not occur at the expense of $D$. dianthicola in French potato agrosystems.

This article is protected by copyright. All rights reserved 
Aside from this national survey, we zoomed at the field level using a more extensive sampling strategy over a four-year period (2013-2016). Along a transect in each of 19 sampled fields, we collected ca. 30 plants with blackleg symptoms and a single isolate was characterized from aerial symptoms of each plant, resulting in the sampling of 548 isolates (Figure 2c). The Pectobacterium populations were present in all the 19 sampled fields. In each of the 19 fields, the null hypothesis that Pectobacterium and Dickeya were randomly distributed was rejected (Chisquared test: $\mathrm{DF}=1 ; \mathrm{p}<0.05)$, meaning that Pectobacterium and Dickeya co-occurred less often than expected under random distribution. The hypothesis that $D$. solani and $D$. dianthicola were randomly distributed along the transect was also rejected in each of the 16 Dickeya-positive fields (Chi-squared tests: $D F=1 ; p<0.05$ ) but one (field \#13; DF= 1; $p=0.24$ ). These field data showed little co-occurrence of taxa at the field levels, both for genera (Dickeya and Pectobacterium) and species ( $D$. solani and $D$. dianthicola). This bias in the symptomatic plants can result from a nonrandom distribution of the taxa among populations in seed tubers or/and soils and surface waters before plant infection, or from competitive exclusion during the infection process of potato plants and tubers. In the following, we focused on the two species $D$. solani and $D$. dianthicola to compare experimentally their fitness in the course of plant and tuber infection, and in particular to test the hypothesis of competitive exclusion.

\subsection{Dickeya dianthicola exhibited a fitness advantage over $D$. solani in potato plants.}

Plant assays were performed in the greenhouse to compare the incidence of blackleg symptoms after inoculation by $D$. dianthicola or $D$. solani on non-wounded potato plants. Five $D$. dianthicola strains (RNS11-47-1-1A, CFBP1888, CFBP2982, CFBP2015, MIE34) and five D. solani strains (3337 = RNS08-23-3-1A, IPO2222, RNS05-1-2A, Ds0432.1, PPO9019) were used in these assays.

In a first assay, each strain was inoculated individually on 15 plants and the number of asymptomatic and symptomatic plants were counted at 70 days post inoculation (dpi). A KruskalWallis test revealed differences between $D$. solani and $D$. dianthicola in terms of symptom incidence $\left(k=7 ; D F=1 ; p=8 \times 10^{-3}\right)$ : $D$. dianthicola was found more virulent than $D$. solani (percentages of symptomatic plants of $61 \% \pm 10$ versus $15 \% \pm 11$; Figure $3 a$ ).

In a second plant assay, the five bacterial cultures (i.e., the five strains) of each species were assembled to constitute two experimental populations and each was inoculated on 15 plants. A mixture of the two species (10 strains) was also inoculated on 15 plants. The assay was duplicated ( $2 \times 15$ plants per treatment). We compared symptom incidence at 70 dpi. KruskalWallis test revealed differences between the three treatments, i.e., $D$. solani strain mixture, $D$. dianthicola strain mixture and species mixture $(k=6.1$; $D F=2 ; p=0.04)$. Pairwise comparison (Post- 
hoc Tukey test) showed that $D$. solani was less virulent than $D$. dianthicola $(F=-2.5 ; p=0.03)$ and the species mixture $(F=1.9 ; p=0.10)$. However, symptom incidence caused by the species mixture did not differ from those of the $D$. dianthicola mixture $(F=-0.5 ; p=0.86)$. Post-hoc Tukey $p$ values and mean value ( \pm SE) of the percentage of symptomatic plants inoculated by the $D$. dianthicola $(53 \% \pm 7)$, D. solani $(23 \% \pm 3)$ and mixed $(47 \% \pm 0)$ populations are presented in the figure $3 \mathbf{b}$. To assess the dynamics of the appearance of symptomatic plants, we also drew a disease progress curve for each replicate (Figure S3a) and compared the areas under disease progress curve (Figure S3b). Pairwise comparisons (Post-hoc Tukey tests) confirmed that $D$. solani was less virulent than $D$. dianthicola $\left(F=-336 ; p<10^{-11}\right)$ and the species mixture $(F=386$; $\left.\mathrm{p}<10^{-11}\right)$.

To evaluate which pathogen had emerged at the beginning of the infection process, hence to identify the most probable causative agent of the observed symptoms, we used qPCR to quantify pathogen loads. When the two species mixtures were inoculated separately, $D$. dianthicola and $D$. solani reached the mean values \pm SE of $2 \times 10^{11} \pm 1 \times 10^{4}$ cells and $3 \times 10^{9} \pm 1 \times 10^{2}$ cells per gram of symptomatic stem tissues, respectively. Among the 14 co-infected plants exhibiting symptoms, eight lesions were randomly chosen for $\mathrm{QPCR}$ quantification of the pathogen abundance: this analysis revealed an excess of $D$. dianthicola with a $\mathrm{Cl}$ median value of $10^{-5}$ (Figure 3c). The eight $\mathrm{Cl}$ values differed from one (Kruskal-Wallis test, $k=9.5 ; D F=1 ; p=2 \times 10^{-3}$ ), meaning that $D$. dianthicola outcompeted $D$. solani in blackleg tissues. Altogether, these data revealed a fitness advantage of $D$. dianthicola in terms of multiplication within lesions in the aerial parts of the host in either the absence or presence of the $D$. solani invader.

\subsection{Dickeya dianthicola exhibited a fitness advantage over $D$. solani in potato plants with wounded roots.}

To evaluate whether plant wounding could benefit to the $D$. dianthicola resident or to the $D$. solani invader, pathogen mixtures were inoculated in pots containing plants with wounded roots. Kruskal-Wallis test revealed differences between the three treatments, i.e., $D$. solani strain mixture, $D$. dianthicola strain mixture and species mixture $\left(k=29.2 ; D F=2 ; p=4.6 \times 10^{-7}\right)$. Pairwise comparisons (post-hoc Tukey tests) showed that the numbers of plants with symptoms differed between inoculations with $D$. solani and $D$. dianthicola $\left(F=-4.5 ; p=1.9 \times 10^{-5}\right)$ and with the mixture

of $D$. dianthicola and $D$. solani $\left(F=6.1 ; p=2.7 \times 10^{-9}\right)$. Mean \pm SE of the percentage of symptomatic plants reached $33 \% \pm 0$ for $D$. solani, $83 \% \pm 10$ for $D$. dianthicola and $93 \% \pm 7$ for a mixture of $D$. dianthicola and $D$. solani, indicating a stronger virulence of $D$. dianthicola as compared to $D$. solani (Figure S4a). Pairwise comparisons (post-hoc Tukey tests) of the areas under the disease curves also confirmed that $D$. solani was less virulent than $D$. dianthicola $\left(F=-386 ; p<10^{-11}\right)$ and 
than a mixture of $D$. dianthicola and $D$. solani $\left(F=386 ; p<10^{-11}\right)$ (Figure $\mathbf{S 3 c}-\mathrm{d}$ ). Because the virulence measured using symptom incidence at the final time point (70 dpi) and area under disease progress curve (from 1 to $70 \mathrm{dpi}$ ) gave similar results when comparing the two pathogens, only the final point of symptom incidence was retained for the analysis of the following plant assays, in order to facilitate the presentation of the data.

In the 27 emerging lesions collected from co-infected plants, qPCR quantification of the pathogens revealed an excess of $D$. dianthicola with a $\mathrm{Cl}$ median value of $10^{-5}$. These $\mathrm{Cl}$ values were different from one (Kruskal-Wallis test: $k=19.5$; $D F=1 ; p=10^{-5}$; Figure $\mathbf{S 3 b}$ ).

Altogether, these experimental data revealed that the resident $D$. dianthicola was more effective at exploiting unwounded and wounded potato host plants than the D. solani invader, i.e. D. dianthicola had a higher fitness regarding this component of its ecological niche.

\subsection{Dickeya solani exhibited a fitness advantage over $D$. dianthicola in potato tubers.}

We compared the $D$. dianthicola and $D$. solani ecological traits in potato tubers. Aggressiveness of the pathogens (i.e., disease severity) was compared using five symptom classes and a disease severity index (DSI) was calculated summarizing the observations of the symptom classes on 10 tubers.

In a first tuber rotting assay, each strain was inoculated separately at $10^{7}$ or at $10^{5} \mathrm{CFU}$ on each set of 10 tubers. The two different inoculum loads allowed comparing the capacity of the pathogens to initiate maceration and exploit tubers in two contrasting ecological conditions. The assay was performed five times, so we collected symptom class information from 50 tubers for each bacterial strain and for each inoculation load. Kruskal-Wallis tests revealed differences between the two treatments, $D$. solani and $D$. dianthicola, both at high $(k=3.2 ; D F=1 ; p=0.07)$ or at low $\left(k=43.6 ; D F=1 ; p=4 \times 10^{-11}\right)$ inoculum dose. The $D S I$ values were calculated for each set of 10 tubers and mean values \pm SE across the five replicates reached $63 \pm 13$ for $D$. solani and $47 \pm$ 4 for $D$. dianthicola with an inoculum of $10^{7}$ CFU per tuber (Figure $4 a$ ) and $49 \pm 8$ for $D$. solani and $7 \pm 4$ for $D$. dianthicola with an inoculum of $10^{5} \mathrm{CFU}$ per tuber (Figure $4 \mathrm{~d}$ ).

In a second tuber assay, we used strain mixtures for each of the two species and an assembly of the two species to inoculate 10 tubers for each of $10^{7}$ and $10^{5}$ CFU per tuber. This assay was performed twice. Kruskal-Wallis tests revealed differences between the three treatments, i.e., $D$. solani, $D$. dianthicola and the species mixture both at a high $\left(k=51.3 ; D F=2 ; p=4.2 \times 10^{-11}\right)$ and a low $\left(k=22.4 ; D F=2 ; p=1.3 \times 10^{-5}\right)$ pathogen load. At both bacterial loads, pairwise comparisons (Post-hoc Tukey tests) showed that aggressiveness was different between $D$. solani and $D$. dianthicola (at a high load: $F=2.7 ; p=0.04$; at a low load: $F=8.6 ; p<10^{-11}$ ). Aggressiveness also differed between $D$. solani and the species mixture (at a high load: $F=2.9 ; p=0.01$; at a low load:

This article is protected by copyright. All rights reserved 
$F=2.2 ; p=0.07)$. A DSI value was calculated for each set of 10 tubers. Mean value \pm SE of the DSI values reached $83 \pm 0$ (D. solani), $63 \pm 3$ (D. dianthicola) and $63 \pm 8$ (D. solani and $D$. dianthicola) with an inoculum of $10^{7} \mathrm{CFU}$ per tuber (Figure 4b) and $69 \pm 14$ (D. solani), $16 \pm 6$ (D. dianthicola) and $48 \pm 10$ (D. solani and $D$. dianthicola) with an inoculum of $10^{5}$ CFU per tuber (Figure 4e). Altogether, these tuber assays revealed a higher aggressiveness of $D$. solani compared to $D$. dianthicola and to the species mixture.

The qPCR analyses of 10 symptomatic tubers revealed that the abundance of the $D$. solani and $D$. dianthicola strain mixtures ranged from $2 \times 10^{9} \pm 3 \times 10^{9}$ to $9 \times 10^{9} \pm 9 \times 10^{9}$ cells per gram of rotted tissues (mean values $\pm S E$ ). These data indicated no substantial differences in the capacity to exploit tubers in terms of population yield. In co-infection assays, qPCR quantification of the pathogens in 10 symptomatic tubers revealed an advantage in favor of $D$. solani regardless of the initial load $(\mathrm{Cl}$ median value $=5.7$; Figure $\mathbf{4 c}$ and $\mathbf{4 f})$. The $\mathrm{Cl}$ values were different from one (Kruskal-Wallis tests: $k=16.3$; $D F=1 ; p$-value of $5 \times 10^{-5}$ with a high load; $k=7.8 ; D F=1 ; p=0.05$ with a low load).

Overall, tubers constituted potato plant tissues in which $D$. solani initiated symptoms more efficiently (even with a low pathogen load), caused more damage (higher disease severity index) and was more competitive than $D$. dianthicola (outcompeting $D$. dianthicola). Remarkably, the disease severity caused by $D$. solani was decreased in the presence of $D$. dianthicola.

\subsection{Dickeya solani exhibited a fitness advantage over $D$. dianthicola in hyacinths.}

The symptom incidence and competition were further evaluated in hyacinths, that represent important hosts as they constitute a reservoir or/and primary or intermediate host of $D$. solani. We expected a higher symptom incidence and growth advantage of $D$. solani on hyacinth host as compared to $D$. dianthicola. The five $D$. dianthicola and five $D$. solani bacterial strains were inoculated separately on eight plants per strain and the number of symptomatic and asymptomatic plants were counted. At $70 \mathrm{dpi}$, a Kruskal-Wallis test revealed differences between $D$. solani and $D$. dianthicola in terms of symptom incidence $(k=3.03 ; D F=1 ; p=0.08)$ : $D$. solani was found more virulent than $D$. dianthicola; means \pm SE of the percentage of symptomatic plants reached $43 \% \pm 17$ and $23 \% \pm 9$, respectively (Figure S5a).

In co-inoculation assays with species mixtures (Figure S5b), the qPCR quantification of the pathogens in five symptomatic tissues showed that the calculated $\mathrm{Cl}$ were different from one (Kruskal-Wallis test: $k=7.8$; $D F=1 ; p=5 \times 10^{-3}$ ). The $\mathrm{Cl}$ median value of $4 \times 10^{9}$ indicated a high competitive advantage of $D$. solani over $D$. dianthicola in rotted tissues of hyacinths. Bulb plants thus appeared promoting competitive exclusion of $D$. dianthicola by $D$. solani, which would lead to an enrichment in $D$. solani in the bulb plant agrosystems. 


\subsection{Pectate lyases gene expression is higher in Dickeya solani than in D. dianthicola}

The capacity of $D$. solani to initiate rotting with a low bacterial load suggested a differential expression of pectate lyase genes between the two species. To test this hypothesis, the expression levels of the pelA, pelD and pelE genes were quantified by RT-qPCR in the five $D$. solani and five $D$. dianthicola strains grown separately in a rich medium (in the absence of pectin) and in emerging lesions of potato stems and tubers (see a detailed protocol in SM2). The expression of two other virulence genes, $\operatorname{cbs} E$ and $a c s D$, which are not involved in the plant cell wall degradation, but in iron-uptake, were also compared. Four RT-qPCR measurements were performed per condition and gene. For each gene, Kruskal-Wallis tests revealed differences between the six treatments (rich medium, stems and tubers for the two species, considering each strain as a replicate) for the different genes: pelA $\left(k=17.1 ; D F=5 ; p=4 \times 10^{-3}\right)$, pelD $(k=25.1 ; D F=5$; $\left.\mathrm{p}=1 \times 10^{-4}\right)$, pelE $\left(\mathrm{k}=24.9 ; \mathrm{DF}=5 ; \mathrm{p}=1 \times 10^{-4}\right)$, cbsE $\left(\mathrm{k}=20.3 ; \mathrm{DF}=5 ; \mathrm{p}=1 \times 10^{-3}\right)$ and $\operatorname{acs} D(\mathrm{k}=21.3$; $\mathrm{DF}=5 ; \mathrm{p}=7 \times 10^{-4}$ ).

Pairwise post-hoc Tukey tests were used to compare gene expression between $D$. solani and $D$. dianthicola. In the absence of pectin (Figure 5), the pelA $\left(\mathrm{F}=864.3 ; \mathrm{p}<10^{-11}\right)$, pelD $(\mathrm{F}=864.3 ; \mathrm{p}<$ $\left.10^{-11}\right)$ and pelE $\left(\mathrm{F}=2.9 ; \mathrm{p}=4 \times 10^{-3}\right)$ genes were expressed at a higher level in $D$. solani than in $D$. dianthicola. The expression levels of the cbsE $(F=0.377 ; p=0.99)$ and $\operatorname{acs} D(F=-1.0 ; p=0.99)$ genes were not significantly different between species (Figure S6). In the absence of pectin, a higher expression of pectate lyase genes, especially pelE, could be considered as an advantage for initiating a rapid degradation of the plant cell wall polymers.

In symptomatic potato tubers and stems, some remarkable gene expression patterns could be noticed (Figures $\mathbf{5}$ and S6). The expression levels of pelD and pelE were higher in $D$. solani than in $D$. dianthicola (pelD: $F=864.3 ; p<10^{-11}$ in tubers and $F=4.5 ; p=10^{-4}$ in stems; $p e l E: F=4.5 ; p=$ $1.1 \times 10^{-4}$ in tubers and $\mathrm{F}=8.1 ; \mathrm{p}<10^{-11}$ in stems $)$. The pelA $\left(\mathrm{F}=864.3 ; \mathrm{p}<10^{-11}\right)$ and $\operatorname{cbs} E(\mathrm{~F}=3.4$; $p=0.01)$ genes were expressed at higher levels in $D$. solani than in $D$. dianthicola stem tissues, but not in tubers ( $p e l A: F=-0.09 ; p=1$ and $c b s E: F=0.67 ; p=0.99$ ). The acs $D$ expression level did not differ between the two species in diseased tubers $(F=-1.5 ; p=0.9)$ and stems $(F=0.5 p=0.9)$. Overall, in plant symptoms, $D$. solani maintained a higher expression of the virulence genes pelD and pelE compared to $D$. dianthicola.

\subsection{Dickeya solani genomics revealed two vfmB alleles at balanced frequencies}

We used a population genomic approach to investigate additional ecological traits of the $D$. solani invading population. We studied SNPs and InDels in 76 D. solani genomes (Figure S2), 56 of which were assembled after Illumina sequencing in this study, while the 20 others had been 
previously acquired using the same sequencing approach (Khayi et al., 2015). Out of the 76 genomes, 66 exhibited from 2 to 11 SNPs and InDels when compared to the D. solani 3337 reference genome (Figure S2). In the nine others, more than 100 SNPs were observed: some of these SNPs were clustered in regions of the chromosome (RNS13-30-1A, PPO9134, PP09019, RNS13-31-1A, RNS13-48-1A, RNS15-102-1A, RNS07-7-3B) while others were distributed along the genome (RNS05-1-2A and RNS10-105-1A).

Focusing on the 66 genomes exhibiting less than 12 SNPs compared to the strain 3337,45 nonsynonymous variations were identified in whole genomes (Table S6). Two-third of them (30 out of 45) were present in one genome only, while the 15 others were found in at least two genomes. Among these 15, three SNPs were identical in four strains, two others in five strains and one was present in 19 genomes (Table $\mathbf{S} \mathbf{6}$ and Figure 6a). The most balanced frequencies consisted in a thymine $(T)$ in 19 genomes and a cytosine $(C)$ residue in the other 48 genomes at the position 2,930,940 in the reference $D$. solani 3337 genome (Figure 6a). The $C$ to T modification caused a proline $\left(\mathrm{VfmB}_{\text {Pro }}\right.$ allele) to serine $\left(\mathrm{VfmB}_{\text {Ser }}\right.$ allele) change at the position 55 in the $\mathrm{VfmB}$ protein. Such SNPs affecting an amino-acid in the $\mathrm{vfmB}$ gene retained our attention because the Vfm quorum-sensing system is involved in the regulation of some virulence traits in Dickeya (Nasser et al., 2013; Potrykus et al., 2018).

A SNP-based tree of these 67 strains (Figure $6 \mathbf{b}$ ) revealed that the $D$. solani isolates carrying $\mathrm{VfmB}_{\text {Ser }}$ were placed in two different sub-clusters, one with only two $\mathrm{VfmB}_{\mathrm{Ser}} D$. solani strains, EU3296 and Ds0432.1, and the other mainly $\mathrm{VfmB}_{\text {Ser }}$ strains. This observation suggests that the $V_{f m B}$ ser allele could have appeared independently in two different $D$. solani lineages, thus representing convergence. The $\mathrm{VfmB}_{\text {Ser }}$ allele was present in $25 \%$ (19 out of 76 ) of all analyzed D. solani genomes. A similar percentage $(27 \%, 17$ out of 63$)$ was observed when only the French isolates were considered. Using the isolation year of these $63 \mathrm{D}$. solani isolates, we drew the dynamics of the percentage of the $\mathrm{VfmB}_{\text {Ser }}$ alleles, which was found to fluctuate over the past decade (2005-2015) with a peak in 2013-2014 (Figure 6c).

We also investigated the presence of the $\mathrm{VfmB}_{\text {Pro }}$ and $\mathrm{VfmB}_{\text {Ser }}$ alleles in other Dickeya species. The alignments of $\mathrm{VfmB}$ proteins retrieved from genomic databases revealed the occurrence of the $\mathrm{VfmB}_{\text {pro }}$ allele in $D$. dadantii, $D$. chrysanthemi and $D$. dianthicola genomes, while the $\mathrm{Vfm} \mathrm{B}_{\text {Ser }}$ allele was found in $D$. zeae genomes (Figure S7). However, the low number of genomes available let open the possibility of the existence of additional alleles in each of these Dickeya species.

\section{8 $\mathrm{VfmB}_{\text {Ser }}$ strains were more aggressive than $\mathrm{VfmB}_{\text {Pro }}$ strains in soft-rot assays}


The modeling of the $D$. solani $\mathrm{VfmB}_{\text {Pro }}$ and $\mathrm{VfmB}_{\text {Ser }}$ protein structures predicted a conformational difference in the beta-sheet structure of the virulence regulator $\mathrm{VfmB}$ (Figure 7a). This change is explained by the exceptional conformational rigidity of proline that strongly affects secondary structures such as alpha helices and beta sheets. The modeling data prompted us to test whether $\mathrm{VfmB}_{\text {Pro }}-\mathrm{VfmB}_{\mathrm{Ser}}$ variation could be associated with a change in aggressiveness. To compare the aggressiveness of $D$. solani $\mathrm{VfmB}_{\text {Ser }}$ and $\mathrm{VfmB}_{\text {Pro }}$ isolates, we used genomic data to identify a set of 8 isolates carrying either $\mathrm{VfmB}_{\text {Pro }}$ (IPO2222, MIE35, AM3a and 3337) or $\mathrm{VfmB}_{\text {Ser }}$ (Ds0432.1, RNS10-27-2A, Sp1a and M21a), in which the other variations were at different positions (Tables S3 and S4). Soft-rot assays using 10 inoculated potato tubers per strain revealed that the set of the $\mathrm{VfmB}_{\text {Ser }}$ strains was more aggressive (Kruskal-Wallis test; $k=9.5 ; D F=1 ; p=2 \times 10^{-3}$ ) than that of the $V f^{\prime} B_{\text {Pro }}$ strains (Figure $7 \mathbf{b}$ ).

To test the existence of differential expression of virulence genes in tuber tissues, we compared transcriptomes of D. solani IPO2222 ( $\left.\mathrm{VfmB}_{\text {Pro }}\right)$ and Ds0432.1 ( $\left(\mathrm{ffmB}_{\text {Ser }}\right)$ (Figure 7c; Table S7). The expression of four genes found differentially expressed based on transcriptomics (pelE, impC, cytA and budA) was further investigated by RT-qPCR in the same samples used for transcriptomics: they found to be also upregulated (Figure S8). Overall, 170 differentially expressed genes (adjusted p-value $<0.05$ and absolute log2 fold change $>2$ ) were identified by comparative transcriptomics. Most of them (150 genes) were upregulated in Ds0432.1 carrying the $\mathrm{VfmB}_{\text {Ser }}$ allele (Table S8). We studied more specifically 35 well-characterized $D$. solani virulence genes (Raoul des Essarts et al., 2019). We observed an enrichment of these virulence genes among the upregulated genes in D. solani Ds0432.1, while none of them was found among the downregulated genes (Table S9). These upregulated virulence genes in $D$. solani Ds0432.1 included the pectate lyase genes pelB, pelC, peID, pelE and pelL, the protease genes ptrA, prtB and prtC, and the T6SS-related toxin/antitoxin genes hcp (Table S9). These upregulated genes encompassed virulence determinants that are already known to be regulated by the Vfm quorumsensing in D. dadantii (Nasser et al., 2013).

\subsection{Fitness costs and gains associated with VfmB alleles in potato stems and tubers}

We used the same eight isolates carrying either $\mathrm{VfmB}_{\text {Pro }}$ (IPO2222, MIE35, AM3a and 3337) or $\mathrm{VfmB}_{\text {Ser }}$ (Ds0432.1, RNS10-27-2A, Sp1a and M21a) to constitute $\mathrm{VfmB}_{\text {Pro }}$ and $\mathrm{VfmB}_{\text {Ser }}$ experimental populations for comparing their fitness in plant assays.

In soft-rot assays (Figure 8a-b), each of the two experimental populations and their mixture was inoculated on 10 tubers and a symptom class was assigned to each tuber. Two inoculation loads were used $\left(10^{7} \mathrm{CFU}\right.$ or $10^{5} \mathrm{CFU}$ per tuber). This assay was performed in triplicate $(3 \times 10$ tubers per treatment). Kruskal-Wallis tests revealed differences between the three treatments, i.e., 
inoculation by $D$. solani $\mathrm{VfmB}_{\text {Pro, }}$ D. solani $\mathrm{VfmB}_{\text {Ser }}$ and their mixture, and the differences were significant at both high $\left(k=17.6 ; D F=2 ; p=1.5 \times 10^{-4}\right)$ and low $(k=7.2 ; D F=2 ; p=0.03)$ pathogen loads. Whatever the bacterial load, pairwise comparisons (Post-hoc Tukey tests) showed that aggressiveness (i.e. symptom severity) was different between $D$. solani $\mathrm{VfmB}$ Pro and $D$. solani $\mathrm{VfmB}_{\text {Ser }}$ (at a high load: $\mathrm{F}=4.6 ; \mathrm{p}=9.5 \times 10^{-6}$; at a low load: $\mathrm{F}=0.8 ; \mathrm{p}=0.01$ ). The $D$. solani $\mathrm{VfmB} \mathrm{B}_{\mathrm{Ser}}$ population was more aggressive than the $D$. solani $\mathrm{VfmB}_{\text {Pro }}$ population (DSI mean value $\pm \mathrm{SE}$ of $92 \pm 2$ for $V f m B_{\text {Ser }} v s 73 \pm 3$ for $V \mathrm{fmB}_{\text {Pro }}$ at a high load and $45 \pm 2$ for $V \mathrm{fmB}_{\text {Ser }}$ vs $26 \pm 9$ for $\mathrm{VfmB}_{\text {Pro }}$ at a low load; Figure $\mathbf{8 a - b}$ ). $\mathrm{VfmB}_{\text {Ser }} D$. solani strains thus more efficiently damaged tubers than $\mathrm{VfmB}_{\mathrm{Pro}} D$. solani strains.

When the $V f m B_{\text {Ser }}$ and $V \mathrm{fmB}_{\text {Pro }}$ D. solani experimental populations were co-infected in tubers, shotgun sequencing of the pathogens recovered from symptoms allowed to calculate $\mathrm{Cl}$ values (Figure 8a-b). With a load of $10^{7} \mathrm{CFU}$ per tuber, the $\mathrm{Cl}$ values (8 values; median $=1.3$ ) were not different from one (Kruskal Wallis test; $k=0.8$; $D F=1 ; p=0.4$ ). With a load of $10^{5} \mathrm{CFU}$ per tuber, the $\mathrm{Cl}$ values ( 3 values; median $=2$ ) differed from one (Kruskal Wallis test; $k=4.4 ; D F=1 ; p=$ 0.04). In tubers, the $\mathrm{VfmB}_{\text {Pro }}$ allele thus appeared neutral or to confer a moderate advantage as compared to the $\mathrm{VfmB}_{\mathrm{Ser}}$ allele.

In blackleg symptom assays (Figure $8 \mathrm{c}$ ), the $D$. solani $\mathrm{VfmB}_{\text {Pro }}$ and $D$. solani $\mathrm{VfmB} \mathrm{B}_{\text {Ser }}$ experimental populations and their mixture were inoculated on non-wounded plants and the number of symptomatic and asymptomatic plants were counted. The assay was performed in triplicate: two assays with 25 plants and one assay with 15 plants per treatment. Kruskal-Wallis tests revealed no difference between the three treatments, i.e., the $D$. solani $V f m B_{\text {Pro }}$ strain mixture, the $D$. solani $\mathrm{VfmB}_{\text {Ser }}$ strain mixture and their mixture $(k=0.29 ; \mathrm{DF}=2 ; p=0.86)$. Shotgun sequencing of bacteria recovered from symptoms allowed calculation of $\mathrm{Cl}$ values in 16 coinfected plants (Figure 8c). These $\mathrm{Cl}$ values were different from one (Kruskal-Wallis test; $\mathrm{k}=6.6$; $\mathrm{DF}=1 ; \mathrm{p}=910^{-3}$ ) with a median $\mathrm{Cl}$ of 14 , showing a fitness advantage of the $\mathrm{VfmB}$ Pro allele in emerging lesions in the stems.

Altogether, the plant assays indicated that the $\mathrm{VfmB}_{\text {Ser }}$ allele was associated to a higher aggressiveness in the rotted tubers compared to the $\mathrm{VfmB}_{\text {Pro }}$ allele. This suggested that the $\mathrm{VfmB}_{\text {Ser }}$ allele could more efficiently initiate maceration of tubers in the field and storage conditions. In contrast, the $\mathrm{VfmB}_{\text {Ser }}$ allele seemed to confer a lower competitive fitness than the $V_{f m B} B_{\text {Pro }}$ allele when $D$. solani proliferates in rotted tubers and, particularly, in stem symptoms. Such opposite gains across different conditions could contribute to the maintenance of balanced frequencies of the two alleles in $D$. solani populations.

\section{Discussion}


Two successive invasions of Dickeya potato pathogens occurred in Europe: first $D$. dianthicola in the middle of the $20^{\text {th }}$ century, this pathogen being now considered as endemic, and second, $D$. solani at the beginning of the $21^{\text {st }}$ century. Dickeya solani and D. dianthicola cause similar symptoms on potato plants, so that they are expected to compete for the same resources. Over ten years, our epidemiologic surveys revealed a successful establishment of $D$. solani together with a maintenance of $D$. dianthicola. Our experiments contributed to explain such puzzling coexistence by contrasted advantages in different parts of the plants and therefore partially different ecological niches.

By comparing ecological traits of $D$. solani and $D$. dianthicola, using multiple strains representative of the population diversity, we indeed found contrasted behaviors in these species with respect to aerial parts versus tubers of $S$. tuberosum. The resident $D$. dianthicola more efficiently exploited host stems after an inoculation of the wounded and unwounded roots than did the invader $D$. solani, i.e., causing higher symptom incidence, and exhibiting greater proliferation and relative fitness in competition assays. In contrast, $D$. solani more efficiently initiated the rotting process (especially at a low bacterial inoculum) and outcompeted $D$. dianthicola in tubers, with potential consequences on tubers in the fields and during storage. Hence, our assays suggest that infected tubers could facilitate the entry and establishment of the $D$. solani invader into the potato agrosystems. A contrasting defense response of potato foliage and tuber against Phytophtora infestans has been reported (Gao \& Bradeen, 2016). In addition to the intrinsic characteristics of $D$. solani and $D$. dianthicola, an organ-specific response of the plant defense system could similarly also contribute to their difference in behavior.

The capacity of $D$. solani to cause rotting at a lower bacterial load than $D$. dianthicola is likely at least partly due to a higher expression of the pel enzymes, especially PelE that acts as an initiator of the plant cell wall degradation (Duprey et al., 2016). By comparing the expression of some pel genes in emerging lesions in potato stems and tubers, we observed a higher expression of the virulence genes peID and pelE in $D$. solani compared to $D$. dianthicola. Remarkably, by comparing pel genes in several Dickeya species, Duprey et al. (2016) showed that D. solani evolved specific regulatory sequences that contributes to a different expression level of the pelD and pelE genes compared to $D$. dianthicola. The pelA gene is truncated in $D$. dianthicola (Duprey et al., 2016; Raoul des Essarts et al., 2019), but full-length and expressed in D. solani (as shown in this study), reinforcing their virulence arsenal. Other metabolic traits could also contribute to the fitness advantage of $D$. solani for exploiting tubers: comparative transcriptomics revealed a higher expression of the glyoxylate shunt in $D$. solani than in $D$. dianthicola, a pathway that contributes to exploit alternative carbon sources when sugar availability is low (Raoul des Essarts et al., 2019). 
Beside the tripartite interaction between potato plant host, $D$. solani and $D$. dianthicola, additional environmental (soil and climate) and biological factors (microbiota including Pectobacterium species, other hosts and non-host plants) may facilitate or limit $D$. solani establishment (Charkowsky 2018; Shyntum et al., 2019; Toth et al., 2011). We showed that $D$. solani efficiently outcompeted $D$. dianthicola in hyacinths, in line with the epidemiologic data supporting the bulb plants as potential intermediate hosts with an important role in the recurrent invasion of potato agrosystems (Chen, Zhang, \& Chen, 2015; Slawiak et al., 2009; van der Wolf et al., 2014). Prophylactics may allow circumventing the propagation of $D$. solani from an agrosystem to another. Competition and facilitation processes have been well studied in pathogenic fungi (AlNaimi, Garrett, \& Bockus, 2005; Abdullah et al., 2017; Gladieux et al., 2015; Zhan \& McDonald, 2013). Another factor that could reduce competition between the two Dickeya species is their natural low abundance in soils and surface waters, as well as in seed tubers that are subjected to prophylactic diagnosis. A small population size belonging to a single species could proliferate in a plant individual without any interactions with another Dickeya or Pectobacterium pathogen species. These effects linked to population size and dispersal are expected to delay invasion. In line with this hypothesis, the observed slow increase of the percentage of Dickeya-positive fields over one decade (2004-2015) suggested that the Dickeya invasion was still ongoing. Among the emerging literature on microbial invasion, the $D$. solani pathogen appears as a good example illustrating how different ecological components (here, the plant host and a resident pathogen) should be considered to understand the biological invasion by a bacterial pathogen (Cadotte et al., 2018; Germain, Mayfield, \& Gilbert, 2018).

The genome sequencing data indicating low diversity in $D$. solani suggest a bottleneck during introduction in Europe. The dispersal modes (either horizontally via soil, surface water, insects and some agricultural practices or vertically by asymptomatically contaminated seed tubers) could contribute to further bottlenecks in D. solani (Charkowsky 2018; Toth et al., 2011). Selection on some genes may also have further reduced genetic diversity through selective sweeps across the whole genomes as these bacteria are clonal. Allelic changes related to quorum-sensing systems were already observed in different plant and animal pathogens along the host-colonization process (Feltner et al., 2016; Guidot et al., 2014; Tannières, Lang, Barnier, Shykoff, Faure, 2017), indicating that balancing selection contributed to maintain their variability. Remarkably, we observed balanced frequencies of two alleles in the VfmB protein involved in the Vfm-type quorum-sensing in Dickeya bacteria. The chemical structure of the Vfm signal is not known, but it is known to be involved in tuber rotting and upregulation of some virulence genes including pectate lyases (pel genes), proteases (prt genes) and cellulases (cel genes) (Nasser et al., 2013; Potrykus et al., 2018). Using tuber assays, we observed a slightly higher 
aggressiveness in $\mathrm{VfmB}_{\text {Ser }}$ strains compared to $\mathrm{VfmB}_{\text {Pro }}$ strains. Using transcriptomics of $D$. solani pathogens recovered from tuber symptoms, we confirmed an enrichment of upregulated genes, including pel and prt genes, in the $\mathrm{VfmB}_{\text {Ser }}$ strain Ds0432.1 compared to $\mathrm{VfmB}_{\text {Pro }}$ strain IPO2222. Because of the role of the $\mathrm{VfmB}$ protein in Vfm signaling is not completely elucidated, it was premature to go deeper into the mechanistic characterization of the $\mathrm{VfmB} \mathrm{B}_{\mathrm{Ser}}$ and $\mathrm{Vfm} \mathrm{B}_{\text {Pro }}$ alleles. In a near future, the reconstruction of allelic mutants and comparison with the wild strain with otherwise the same genetic background should help characterizing the role of $\mathrm{VfmB}$ in $\mathrm{Vfm}$ quorum-sensing and ecology of $D$. solani.

In this work, we strongly suggest that the $\mathrm{VfmB}_{\text {Ser }}$ strains are more aggressive than the $\mathrm{VfmB}$ Pro strains in potato tubers, but not more virulent in potato stem assays, and that the $\mathrm{VfmB} \mathrm{B}_{\text {Ser }}$ strains were less competitive than the $\mathrm{VfmB}_{\text {Pro }}$ strains in tuber and stem symptoms. Such an environment-dependent advantage of $\mathrm{VfmB}_{\text {ser }}$ and $\mathrm{VfmB}_{\text {Pro }}$ alleles in plant infection assays predicts balancing selection in natural populations. The $\mathrm{VfmB}_{\text {ser }}$ allele could thus provide an advantage when competition is reduced at the beginning of the establishment in potato agrosystems and under a high dispersal condition. In contrast, $\mathrm{VfmB}_{\text {ser }}$ could be outcompeted by $\mathrm{VfmB}_{\text {Pro }}$ when $D$. solani is already established. Our epidemiologic data revealed up and down variation of $\mathrm{VfmB}_{\text {ser }}$ relative abundance in field populations over the past decade, in agreement with an increase of competition between $D$. solani $\mathrm{VfmB}$ alleles in the more recent sampling year. There is a very low number of SNPs/InDels $(<12)$ in the genomes beyond those in Vfm, however, functional genetics will be required to fully validate the causal role of the Vfm alleles.

In conclusion, this study using complementary approaches, multiple strains representative of the population diversity and multiple ecological conditions, brings novel insights allowing a better understanding of the pattern and causes of the $D$. solani invasion into potato production agrosystems, and the reasons why $D$. dianthicola nevertheless persisted. More broadly, this study contributes to our understanding the ecological determinants of pathogen invasion and of the conditions for the maintenance of endemic competitors.

\section{ACKNOWLEDGEMENTS}

PB was supported by a PhD grant from Université Paris-Sud (Université Paris-Saclay), KR was supported by a PhD grant from FN3PT/inov3PT with the support of ANRT (N²016/0800). KGC thanks the financial support from the University of Malaya (FRGS grant FP022-2018A and HIR grant $\mathrm{H}-50001-\mathrm{A000027)}$. This work has benefited from the facilities and expertise of the high throughput sequencing platform and the plant culture facilities at I2BC (Gif-sur-Yvette). This work was supported by CNRS (SE2016-2017-2018), French Federation of Seed Potato Growers 
(FN3PT/inov3PT), Agence Nationale de la Recherche (ANR-15-CE21-0003) and LabEx Saclay Plant Sciences-SPS (ANR-10-LABX-0040-SPS).

\section{REFERENCES}

Abdullah, A. S., Moffat, C. S., Lopez-Ruiz, F. J., Gibberd, M. R., Hamblin, J., \& Zerihun, A. (2017). Host-multi-pathogen warfare: pathogen interactions in co-infected plants. Frontiers in Plant Science, 8,1806.

Al-Naimi F. A., Garrett, K. A., \& Bockus, W. W. (2005). Competition, facilitation, and niche differentiation in two foliar pathogens. Oecologia, 143, 449-457.

Bellieny-Rabelo, D., Tanui, C.K., Miguel, N., Kwenda, S., Shyntum, D.Y., \& Moleleki, L.N. (2019). Transcriptome and comparative genomics analyses reveal new functional insights on key determinants of pathogenesis and interbacterial competition in Pectobacterium and Dickeya spp. Applied and Environmental Microbiology, 85, pii:e02050-18.

Burns, J. H., Murphy, J. E., \& Zheng, Y. L. (2018). Tests of alternative evolutionary models are needed to enhance our understanding of biological invasions. New Phytologist, 222, 701707.

Cadotte, M. W., Campbell, S. E., Li, S. P., Sodhi, D. S., \& Mandrak, N. E. (2018). Preadaptation and naturalization of nonnative species: Darwin's two fundamental insights into species invasion. Annual Reviews of Plant Biology, 69, 661-684.

Charkowsky, A.O. (2018). The changing face of bacterial soft-rot diseases. Annual Reviews of Phytopathology, 56, 269-288.

Chen, X. F., Zhang H. L., \& Chen, J. (2015). First report of Dickeya solani causing soft-rot in imported bulbs of Hyacinthus orientalis in China. Plant Disease, 99,155.

Crépin, A., Barbey, C., Beury-Cirou, A., Hélias, V., Taupin, L., Reverchon, S., ... Latour X. (2012). Quorum sensing signaling molecules produced by reference and emerging soft-rot bacteria (Dickeya and Pectobacterium spp.). PLoS One, 7, e35176.

Czajkowski, R., de Boer, W. J., van der Zouwen, P. S., Kastelein, P., Jafra, S., de Haan, E.G., ... van der Wolf, J. M. (2013). Virulence of 'Dickeya solani' and Dickeya dianthicola biovar-1 and -7 strains on potato (Solanum tuberosum). Plant Patholology, 62, 597-610.

Czajkowski, R., de Boer, W. J., Velvis, H., \& van der Wolf, J. M. (2010). Systemic colonization of potato plants by a soilborne, green fluorescent protein-tagged strain of Dickeya sp. biovar 3. Phytopathology, 100, 134-142.

Duprey, A., Nasser, W., Léonard, S., Brochier-Armanet, C., \& Reverchon, S. (2016). Transcriptional start site turnover in the evolution of bacterial paralogous genes - the pelEpelD virulence genes in Dickeya. FEBS Journal, 283, 4192-4207. 
Facon, B., Genton, B. J., Shykoff, J., Jarne, P., Estoup, A., \& David, P. (2006). A general ecoevolutionary framework for understanding bioinvasions. Trends in Ecology \& Evolution, 21, 130-135.

Feltner, J. B., Wolter, D. J., Pope, C. E., Groleau, M. C., Smalley, N., E., Greenberg, E. P., ... Dandekar, A. A. (2016). LasR variant cystic fibrosis isolates reveal an adaptable quorumsensing hierarchy in Pseudomonas aeruginosa. mBio 7(5): e01513-16.

Francisco, A. P., Vaz, C., Monteiro, P. T., Melo-Cristino, J., Ramirez, M., \& Carriço, J. A. (2012). PHYLOViZ: Phylogenetic inference and data visualization for sequence based typing methods. BMC Bioinformatics, 13, 87.

Gao, L., \& Bradeen, J. M. (2016). Contrasting potato foliage and tuber defense mechanisms against the late blight pathogen Phytophthora infestans. PLoS One, 11(7):e0159969.

Germain, R. M., Mayfield, M. M., \& Gilbert, B. (2018). The 'filtering' metaphor revisited: competition and environment jointly structure invasibility and coexistence. Biology Letters, 14, 20180460.

Gladieux, P., Feurtey, A., Hood, M. E., Snirc, A., Clavel, J., Dutech, C., ... Giraud, T. (2015) The population biology of fungal invasions. Molecular Ecology, 24, 1969-1986.

Golanowska, M., Potrykus, M., Motyka-Pomagruk, A., Kabza, M., Bacci, G., Galardini, M., ... Lojkowska, E. (2018). Comparison of highly and weakly virulent Dickeya solani strains, with a view on the pangenome and panregulon of this species. Frontiers in Microbiology, 9,1940 .

Guidot, A., Jiang, W., Ferdy, J.B., Thébaud, C., Barberis, P., Gouzy, J., \& Genin, S. (2014). Multihost experimental evolution of the pathogen Ralstonia solanacearum unveils genes involved in adaptation to plants. Molecular Biology and Evolution, 31, 2913-2928.

Hélias, V., Hamon, P., Huchet, E., Wolf, J. V. D., \& Andrivon, D. (2012). Two new effective semiselective crystal violet pectate media for isolation of Pectobacterium and Dickeya. Plant Pathology, 61, 339-345.

Kelley, L. A., Mezulis, S., Yates, C. M., Wass, M. N., \& Sternberg, M. J. E. (2015). The Phyre2 web portal for protein modeling, prediction and analysis. Nature Protocols, 10, 845-858.

Khayi, S., Blin, P., Pédron, J., Chong, T. M., Chan, K. G., Moumni, M., .. Faure, D. (2015). Population genomics reveals additive and replacing horizontal gene transfers in the emerging pathogen Dickeya solani. BMC Genomics, 16, 788.

Kinnunen, M., Dechesne, A., Proctor, C., Hammes, F., Johnson, D., Quintela-Baluja, M., ... Smets BF. (2016). A conceptual framework for invasion in microbial communities. ISME Journal, 10, 2773-2775.

This article is protected by copyright. All rights reserved 
Kumar, S., Stecher, G., \& Tamura, K. (2016). MEGA7: Molecular evolutionary genetics analysis version 7.0 for bigger dataset. Molecular Biology and Evolution, 33, 1870-1874.

Laurila, J., Ahola, V., Lehtinen, A., Joutsjoki, T., Hannukkala, A., Rahkonen, A., \& Pirhonen, M. (2008). Characterization of Dickeya strains isolated from potato and river water samples in Finland. European Journal of Plant Pathology, 122, 213-225.

Léonard, S., Hommais, F., Nasser, W., \& Reverchon, S. (2017). Plant-phytopathogen interactions: bacterial responses to environmental and plant stimuli. Environmental Microbiology, 19, 1689-1716.

Litchman, E. (2010). Invisible invaders: non-pathogenic invasive microbes in aquatic and terrestrial ecosystems. Ecology Letters, 13, 1560-1572.

MacDougall, A. S., Gilbert, B., \& Levine, J. M. (2009). Plant invasions and the niche. Journal of Ecology, 97, 609-615.

Mallon, C. A., Elsas, J. D. V., \& Salles, J. F. (2015). Microbial invasions: the process, patterns, and mechanisms. Trends in Microbiology, 23, 719-729.

Nasser, W., Dorel, C., Wawrzyniak, J., Van Gijsegem, F., Groleau, M. C., Déziel, E., \& Reverchon, S. (2013). Vfm a new quorum sensing system controls the virulence of Dickeya dadantii. Environmental Microbiology, 15, 865-880.

Oulghazi, S., Khayi, S., Lafkih, N., Massaoudi Y, El Karkouri A, El Hassouni M, ... Moumni, M. (2017). First report of Dickeya dianthicola causing blackleg disease on potato in Morocco. Plant Disease, 101, 1671.

Parkinson, N., Stead, D., Bew, J., Heeney, J., Tsror Lahkim, L., \& Elphinstone, J. (2009). Dickeya species relatedness and clade structure determined by comparison of recA sequences. International Journal of Systematic and Evolutionary Microbiology, 59, 2388-2393.

Patel, N., Baldwin, A. C., Patel, R. D., Kobayashi, D. Y., \& Wyenandt, C. A. (2019). First report of Dickeya dianthicola causing blackleg and soft-rot on potato (Solanum tuberosum) in New Jersey, U.S.A. Plant Disease, 103, 146.

Perkins, L. B., Leger, E. A., \& Nowak, R. S. (2011). Invasion triangle: an organizational framework for species invasion. Ecology and Evolution, 1, 610-625.

Pérombelon, M. C. M. (2002). Potato diseases caused by soft-rot erwinias: an overview of pathogenesis. Plant Pathology, 51, 1-12.

Potrykus, M., Golanowska, M., Hugouvieux-Cotte-Pattat, N., \& Lojkowska, E. (2014). Regulators involved in Dickeya solani virulence, genetic conservation and functional variability. Molecular Plant Microbe Interactions, 27, 700-711.

This article is protected by copyright. All rights reserved 
Potrykus, M., Golanowska, M., Sledz, W., Zoledowska, S., Motyka, A., Kolodziejska, A., ... Lojkowska, E. (2016). Biodiversity of Dickeya spp. isolated from potato plants and water sources in temperate climate. Plant Disease, 100, 408-417.

Potrykus, M., Hugouvieux-Cotte-Pattat, N., \& Lojkowska, E. (2018). Interplay of classic Exp and specific Vfm quorum sensing systems on the phenotypic features of Dickeya solani strains exhibiting different virulence levels. Molecular Plant Pathology, 19, 1238-1251.

Raoul des Essarts, Y., Cigna, J., Quêtu-Laurent, A., Caron, A., Munier, E., Beury-Cirou, A., ... Faure, D. (2015). Biocontrol of the potato blackleg and soft rot diseases caused by Dickeya dianthicola. Applied and Environmental Microbiology, 82, 268-278.

Raoul des Essarts, Y., Pédron, J., Blin, P., Van Dijk, E., Faure, D., \& Van Gijsegem, F. (2019). Common and distinctive adaptive traits expressed in Dickeya dianthicola and Dickeya solani pathogens when exploiting potato plant host. Environmental Microbiology, 21, 10041018.

Reynolds, C.R., Islam, S. A., Sternberg, M. J. E. (2018). EzMol: A Web Server Wizard for the Rapid Visualization and Image Production of Protein and Nucleic Acid Structures. Journal of Molecular Biology, 430, 2244-2248.

Sarfraz, S., Riaz, K., Oulghazi, S., Cigna, J., Alam, M. W., Dessaux, Y., \& Faure, D. (2018). First report of Dickeya dianthicola causing blackleg disease on potato plants in Pakistan. Plant Disease, 102, 2027.

Shyntum, D. Y., Nkomo, N. P., Shingange, N. L., Gricia, A. R., Bellieny-Rabelo, D., \& Moleleki, L. N. (2019). The Impact of Type VI secretion system, bacteriocins and antibiotics on bacterial competition of Pectobacterium carotovorum subsp. brasiliense and the regulation of carbapenem biosynthesis by iron and the ferric-uptake regulator. Frontiers in Microbiology, 10, 2379. doi: 10.3389/fmicb.2019.02379

Sławiak, M., van Beckhoven, J. R. C. M., Speksnijder, A. G. C. L., Czajkowski, R., Grabe, G., \& van der Wolf, J. M. (2009). Biochemical and genetical analysis reveal a new clade of biovar 3 Dickeya spp. strains isolated from potato in Europe. European Journal of Plant Pathology, 125, 245-261.

Tannières, M., Lang, J., Barnier, C., Shykoff, J. A., \& Faure, D. (2017). Quorum-quenching limits quorum-sensing exploitation by signal-negative invaders. Scientific Reports, 7, 40126. doi: 10.1038/srep40126.

Toth, I. K., van der Wolf, J. M., Saddler, G., Lojkowska, E., Hélias, V., Pirhonen, ... Elphinstone, J. G. (2011). Dickeya species an emerging problem for potato production in Europe. Plant Pathology, 60, 385-399. 
van der Wolf, J. M., Nijhuis, E. H., Kowalewska, M. J., Saddler, G. S., Parkinson, N., Elphinstone, J. G., ... Manulis, S. (2014). Dickeya solani sp. nov., a pectinolytic plant-pathogenic bacterium isolated from potato (Solanum tuberosum). International Journal of Systematic and Evolutionary Microbiology, 64, 768-774.

Wright, D., Bwye, A., Banovic, M., Baulch, J., Wang, C., Hair, S., ... Kehoe, M. (2018). First report of Dickeya dianthicola in potatoes in Australia. Plant Disease, 102, 2029.

Zhan, J., \& McDonald, B. A. (2013). Experimental measures of pathogen competition and relative fitness. Annual Reviews of Phytopathology, 51, 131-153.

\section{DATA ACCESSIBILITY}

DNA sequences: Genbank accessions VYSC00000000; VZQE00000000; VZQF00000000; VZQG00000000; VZQH00000000; VZQI00000000; VZQK00000000; VZQJ00000000; VZQL00000000; VZQM00000000

\section{AUTHOR CONTRIBUTION}

$\mathrm{DF}, \mathrm{PB}, \mathrm{VH}$ conceived experiments; JC, EM, PD, AL, AB and $\mathrm{VH}$ performed field sampling and molecular diagnosis; PB characterized fitness traits in $D$. solani and $D$. dianthicola; PB, KR and EM characterized fitness traits in $D$. solani vfmB variants; PB performed RNA analyses (RTqPCR and transcriptomics); YJ, KWH, KGC, AL and PB prepared DNA samples and perforemd sequencing; SK compared $D$. solani genomes; $\mathrm{PB}, \mathrm{KR}$ and DF performed statistical analyses; DF, TG, PB, VH, SR wrote the manuscript.

\section{CONFLICT OF INTEREST}

No potential conflict of interest was disclosed.

\section{ORCID}

Denis Faure orcid.org/0000-0002-5379-8867 


\section{Figure legends}

Figure 1 Symptoms caused by Dickeya pathogens. Symptoms (white arrows) caused by Dickeya solani and Dickeya dianthicola on potato stems (a) and hyacinths (b). The five symptom classes (0 to 4) used to compare the aggressiveness of Dickeya solani and Dickeya dianthicola on potato tubers (c).

Figure 2 Dickeya and Pectobacterium prevalence from potato fields exhibiting blackleg symptoms. Percentage of Pectobacterium-containing and Dickeya-containing fields (a) and that of $D$. solani-containing fields and $D$. dianthicola-containing fields (b) were calculated each year from 2004 to 2015 (with the exception of 2006). (c) Number and relative abundance of the Pectobacterium, $D$. dianthicola and $D$. solani isolates collected in 19 symptomatic fields sampled from 2013 to 2016 . A hierarchical clustering paired group method delineated four pathogen population groups, i.e. Pectobacterium only (I: 3 fields), D. solani and Pectobacterium (II: 9 fields), D. dianthicola and Pectobacterium (III: 3 fields), and D. dianthicola, D. solani and Pectobacterium (IV: 4 fields).

Figure 3 Symptom incidence and fitness of Dickeya dianthicola and D. solani in potato plants. In a, mean value and standard error (SE) of the percentage (\%) values of plants exhibiting blackleg symptoms, which were measured after inoculation of each of the five $D$. solani strains (3337, IPO2222, RNS05-1-2A, Ds0432.1, PPO9019) and five D. dianthicola strains (RNS11-47-1-1A, CFBP1888, CFBP2982, CFBP2015, MIE34) on 15 plants. The p-value of the Kruskal-Wallis test comparing of the symptomatic classes between the two species is indicated below the graph. In $\mathbf{b}$, mean value and SE between two replicates of the percentages (\%) of plants exhibiting blackleg symptoms which was measured on 15 plants inoculated by $D$. dianthicola and $D$. solani populations and their mixture. The $p$ values of the pairwise comparisons (Post-hoc Tukey tests) of the symptomatic classes are indicated below the graph, when $\mathrm{p} \leq 0.1$. In $\mathbf{c}$, competitive index $(\mathrm{Cl})$ values between $D$. solani and $D$. dianthicola populations were calculated in eight emerging lesions of co-infected plants and revealed a competitive advantage of $D$. dianthicola: the $\mathrm{Cl}$ median $\left(=2.410^{-5}\right)$ is represented by a thick bar; $\mathrm{p}$-values resulting from Kruskal-Wallis tests testing difference from one are indicated. Legend: ${ }^{*}$ for $0.05<p \leq 0.1$; ${ }^{*}$ for $0.01<p \leq 0.05$ and $^{* * *}$ for $p \leq 0.01$.

This article is protected by copyright. All rights reserved 
Figure 4 Aggressiveness and fitness of Dickeya dianthicola and $D$. solani in potato tubers. Rotting assays were conducted by inoculating each tuber by either $10^{7}$ colony-forming units (CFU) (in $\mathbf{a}, \mathbf{b}$ and $\mathbf{c}$ ) or $10^{5} \mathrm{CFU}$ of pathogens (in $\mathbf{d}, \mathbf{e}$ and $\mathbf{f}$ ). In $\mathbf{a}, \mathbf{b}, \mathbf{d}$ and $\mathbf{e}$, each disease severity index (DSI) value was calculated using symptom classes observed on 10 tubers. In a and $\mathbf{d}$, mean values and standard errors (SE) of DSI values were measured for each of the five $D$. solani strains (3337, IPO2222, RNS05-1-2A, Ds0432.1, PPO9019) and five D. dianthicola strains (RNS11-47-1-1A, CFBP1888, CFBP2982, CFBP2015, MIE34) using five (a) and two (d) independent experiments. The p-values of the Kruskal-Wallis tests comparing symptom classes are indicated below the graphs. In $\mathbf{b}$ and $\mathbf{e}$, mean values and standard errors of DSI values were measured twice for each of the experimental populations of $D$. dianthicola, $D$. solani and the mixture of the two. The p-values of the pairwise Tukey tests comparing the symptomatic classes are indicated, when $p \leq 0.1$. In $\mathbf{c}$ and $\mathbf{f}$, the competitive index $(\mathrm{Cl})$ between $D$. solani and $D$. dianthicola populations was calculated in $10 \mathrm{co}-$ infected symptomatic tubers: the median values (indicated by a thick bar) reached 5.7 (c) and 5.8 (f) and the $\mathrm{Cl}$ values were statistically different from one (Kruskal-Wallis test; $p=510^{-5}$ and $p=0.05$ respectively), revealing a competitive advantage of $D$. solani. Legend: * for $0.05<p \leq 0.1$; ${ }^{* *}$ for $0.01<p \leq 0.05$ and $^{* * *}$ for $p \leq 0.01$.

Figure 5 Expression of the pectate lyase genes peIA, peID and pelE. The expression level of the pelA, pelD and pelE genes was evaluated in each of the five $D$. solani strains (3337, IPO2222, RNS051-2A, Ds0432.1, PPO9019) and five D. dianthicola strains (RNS11-47-1-1A, CFBP1888, CFBP2982, CFBP2015, MIE34), grown in three conditions: a rich culture medium in the absence of pectin (exponential growth phase) and symptomatic tubers (at 5 days post infection) and emerging lesions in stems. Relative expression was measured four times and normalized using the $r p o B$ and yafS gene expression. In the graphs, the mean values and standard error (SE) of gene expression from all strains of a given species are indicated, as well as p-values of pairwise comparisons by Tukey tests. Legend: * for $0.05<p \leq 0.1 ;{ }^{* *}$ for $0.01<p \leq 0.05$ and ${ }^{* * *}$ for $p \leq 0.01$.

Figure 6 Population genomics of Dickeya solani. In a, scan of $67 \mathrm{D}$. solani genomes revealed that the genome position 2,930,940, (according the $D$. solani 3337 genome), in the vfmB gene, was the most balanced non-synonymous variation, with alternated $\mathrm{VfmB}_{\text {Pro }}(71 \%)$ and $\mathrm{VfmB}_{\text {Ser }}(29 \%)$ alleles. In b, single nucleotide polymorphism (SNP)-based tree of $D$. solani strains using PHYLOViZ; the name of the French isolates is indicated in blue font; the $\mathrm{VfmB}_{\text {Ser }}$ and $\mathrm{VfmB}_{\text {Pro }}$ strains used in the plant assays are underlined. In c, dynamics of the $\mathrm{VfmB}_{\text {Ser }}$ allele (\%) among the $D$. solani isolates along the sampling period 2005-2015 in France.

This article is protected by copyright. All rights reserved 
Figure 7 Characterization of the VfmB alleles. In a, using the Escherichia coli AidB protein (the PDB accession is 3DJL) as a model, the Phyre2-predicted structure of the $\mathrm{VfmB}$ protein showed conformational differences in the beta-sheet associated with the $\mathrm{VfmB}_{\text {Pro }}$ and $\mathrm{VfmB}$ Ser alleles; the Pro55 and Ser55 positions are in red color in the VfmB representations obtained using the EzMol web server. In b, mean values and standard error (SE) of disease severity index (DSI) values, which were calculated by recording symptom classes on 10 tubers infected by $10^{7} \mathrm{CFU}$ of each of the $D$. solani

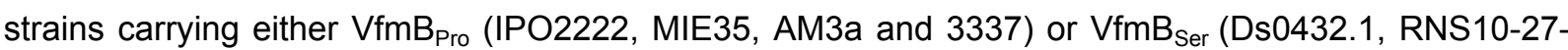
$2 \mathrm{~A}, \mathrm{Sp} 1 \mathrm{a}$ and M21a); the p-value of the Kruskal-Wallis test comparing the four $\mathrm{VfmB} \mathrm{B}_{\text {Pro }}$ and four $\mathrm{VfmB}_{\text {Ser }}$ strains using symptom classes is indicated below the graph. In c, comparative transcriptome of D. solani $3337\left(\mathrm{VfmB}_{\text {Pro }}\right)$ and Ds0432.1 ( $\left.\mathrm{VfmB}_{\text {Ser }}\right)$ recovered from soft-rot lesions in potato tubers; upregulated virulence genes were enriched in $\mathrm{Ds} 0432.1\left(\mathrm{VfmB}_{\mathrm{Ser}}\right)$ as illustrated by the pelB, pelC, pelC, hcpA and prtA genes (closed circles).

Figure 8 Aggressiveness, symptom incidence and fitness assays of two Dickeya solani experimental populations expressing the VfmB alleles. Soft-rot assays were conducted by inoculating each tuber by either $10^{7}$ colony-forming units (CFU) (a) or $10^{5} \mathrm{CFU}$ (b) of the $\mathrm{VfmB}_{\text {Pro }}$ and $\mathrm{VfmB}_{\text {Ser }} D$. solani populations and a mixture of the two. In $\mathbf{a}$ and $\mathbf{b}$, each disease severity index (DSI) value (measuring aggressiveness) was calculated using symptomatic classes observed on 10 tubers; the assays were performed in triplicate. The p-values of the pairwise Tukey tests comparing the symptom classes are indicated, when $p \leq 0.1$. In c, percentage (\%) of plants exhibiting blackleg symptoms was measured on plants inoculated by the $\mathrm{VfmB}_{\text {Pro }}$ and $\mathrm{VfmB}_{\mathrm{Ser}} D$. solani populations and a mixture of the two. These assays measuring symptom incidence were performed in triplicate. KruskalWallis test revealed no difference between the three treatments using symptom classes $(p=0.86)$. In $\mathbf{a}$, b and $\mathbf{c}$, competitive index $(\mathrm{Cl})$ values of $\mathrm{VfmB}_{\text {Pro }}$ and $\mathrm{VfmB}_{\text {Ser }}$ populations were calculated using allele counts based on shotgun sequencing of bacterial populations recovered from co-infected tissues. Median values of $\mathrm{Cl}$ are represented as thick lines and reached 1.7 (8 repeats in a), 2.0 (3 repeats in $\mathbf{b}$ ) and 14.0 (16 repeats in $\mathbf{c}$ ) in the three treatments. Statistical differences between $\mathrm{Cl}$ values and 1 (Kruskal-Wallis test) revealed that neither $D$. solani $V \mathrm{fmB}_{\text {Pro }}$ or $D$. solani $\mathrm{VfmB}_{\mathrm{Ser}}$ had significant advantage in tubers inoculated with a high load $(p=0.3$ in a), but $D$. solani VfmB Pro was more competitive in tubers inoculated with a low load ( $p=0.04$ in $\mathbf{b})$ and in stems $\left(p=9 \times 10^{-3}\right.$ in $\left.\mathbf{c}\right)$. Legend: ${ }^{*}$ for $0.05<p \leq 0.1 ;{ }^{* *}$ for $0.01<p \leq 0.05$ and ${ }^{* * *}$ for $p \leq 0.01$. 
Figure 1

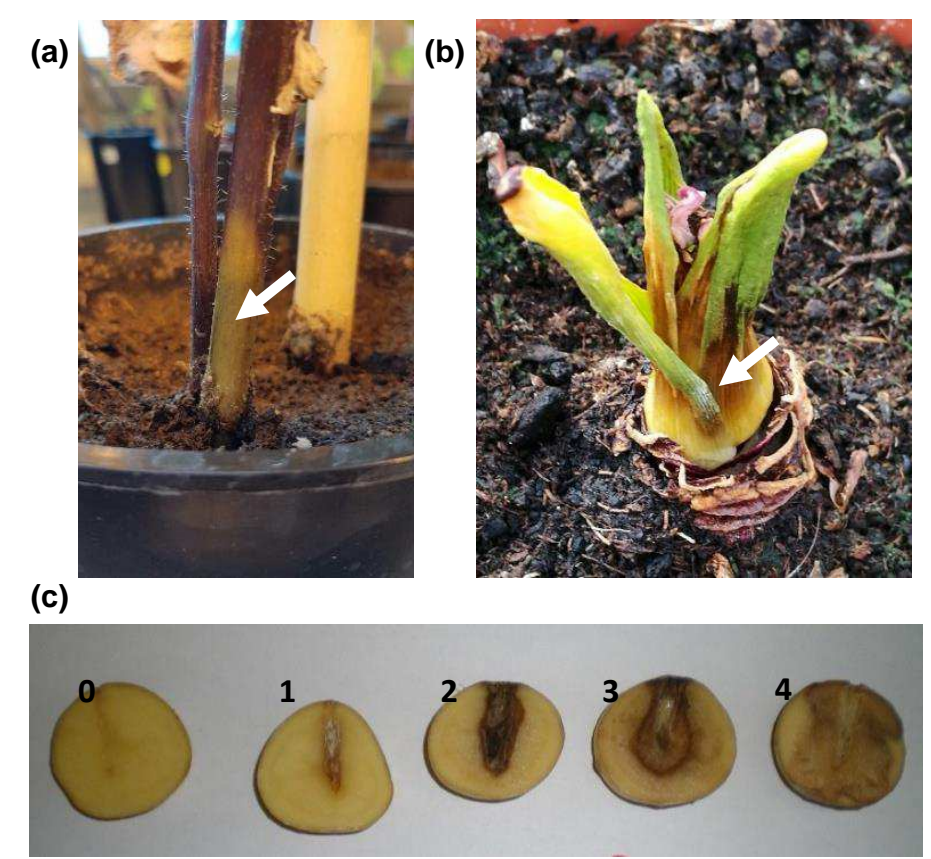

This article is protected by copyright. All rights reserved 


\section{Figure2}

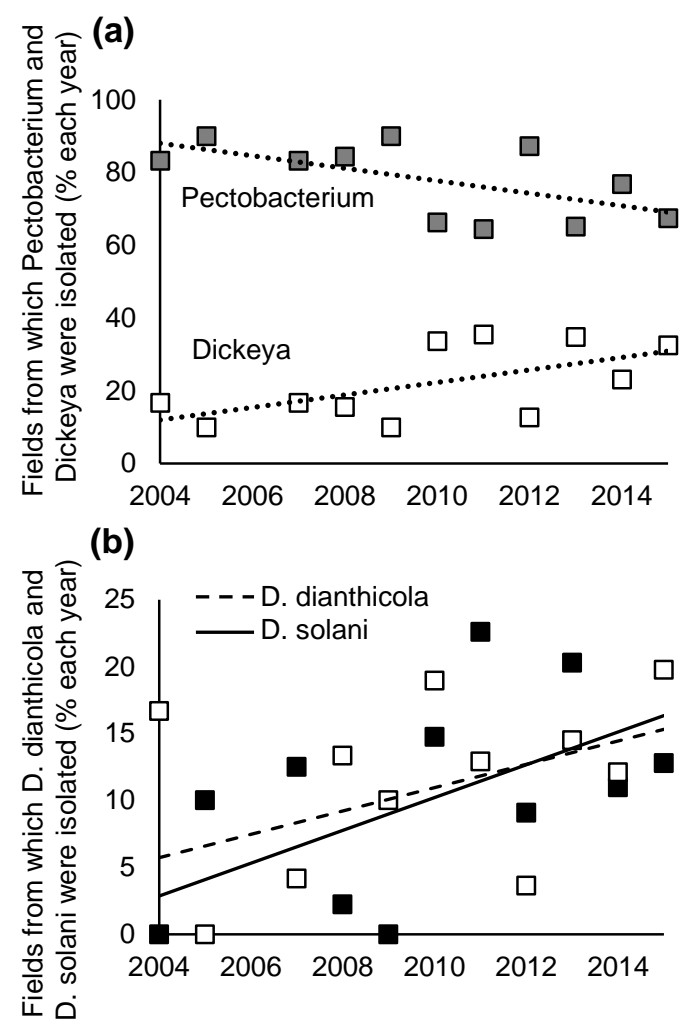

Field code \#

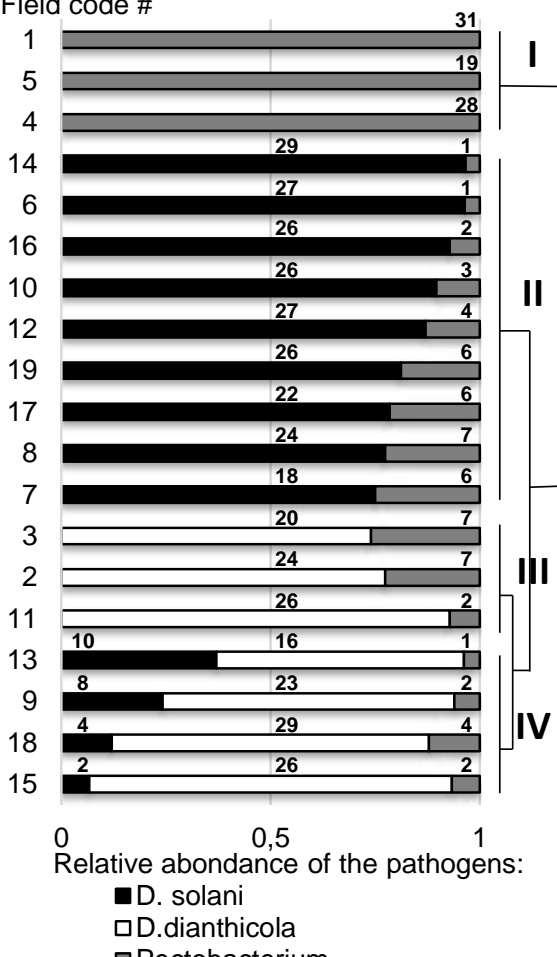

口D.dianthico

$\square$ Pectobacterium 


\section{Figure 3}

(a)

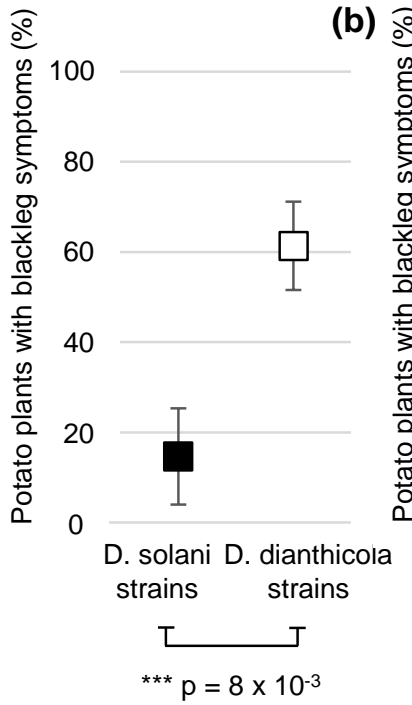

(b) $\stackrel{\circ}{\varrho}$

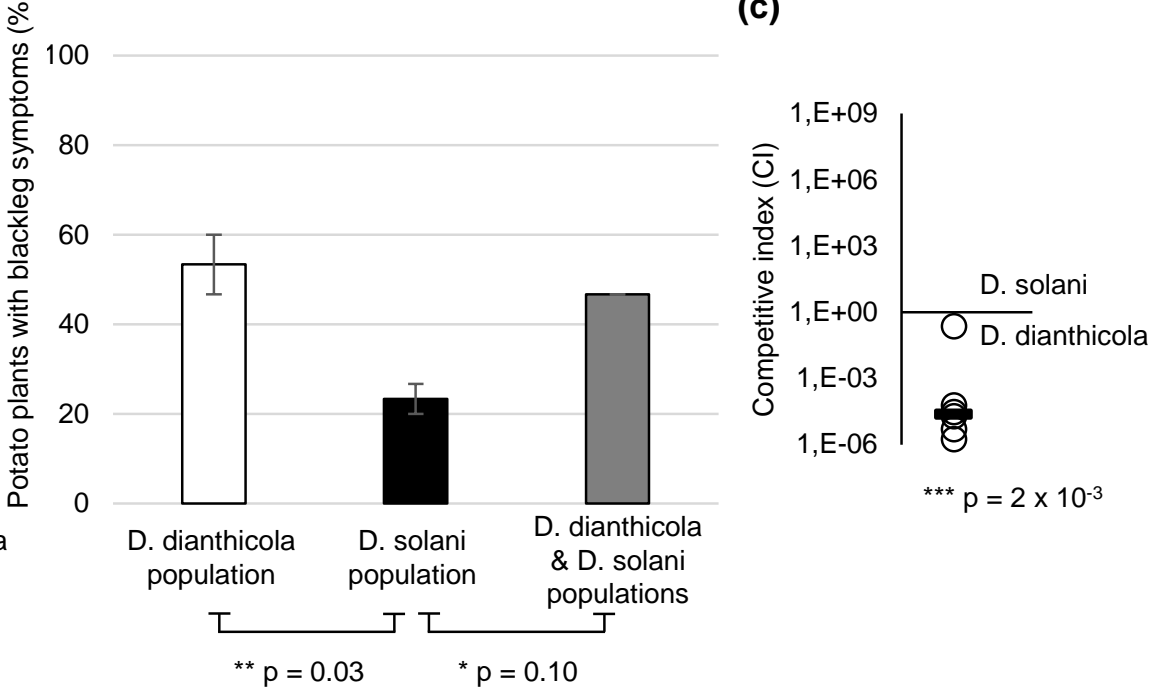

This article is protected by copyright. All rights reserved 


\section{Figure 4}

(a)

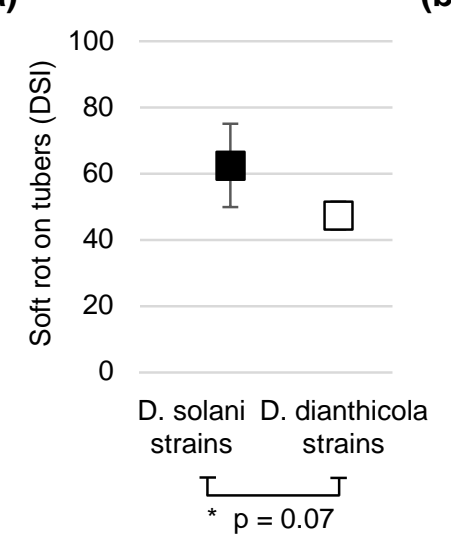

(d)

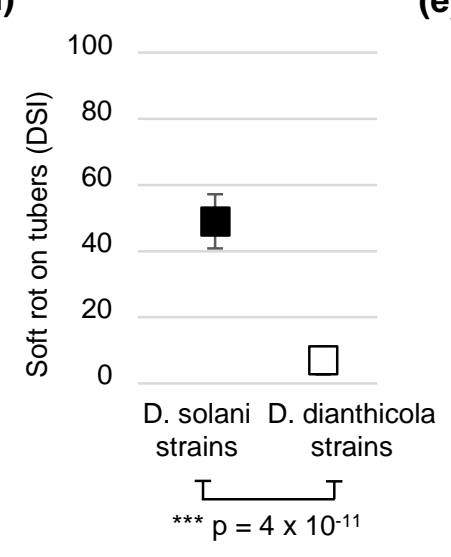

(b)

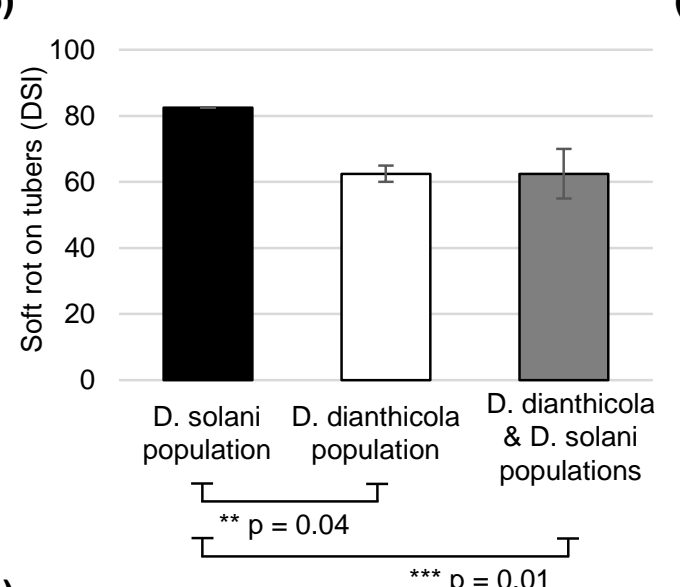

(e)

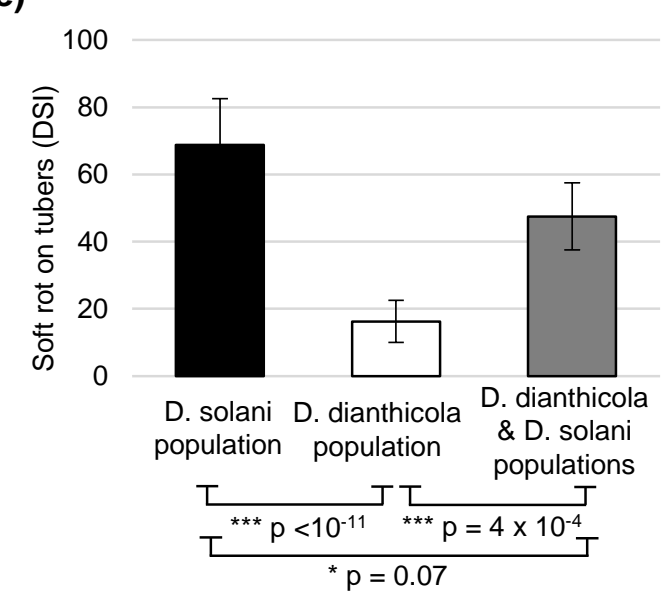

(c)

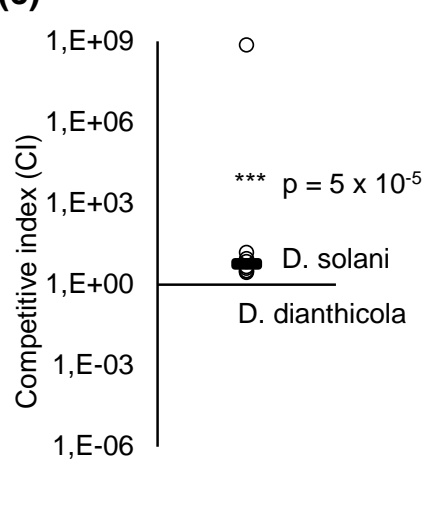

(f)

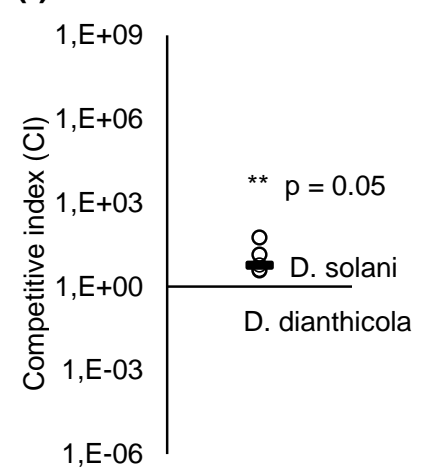




\section{Figure 5}

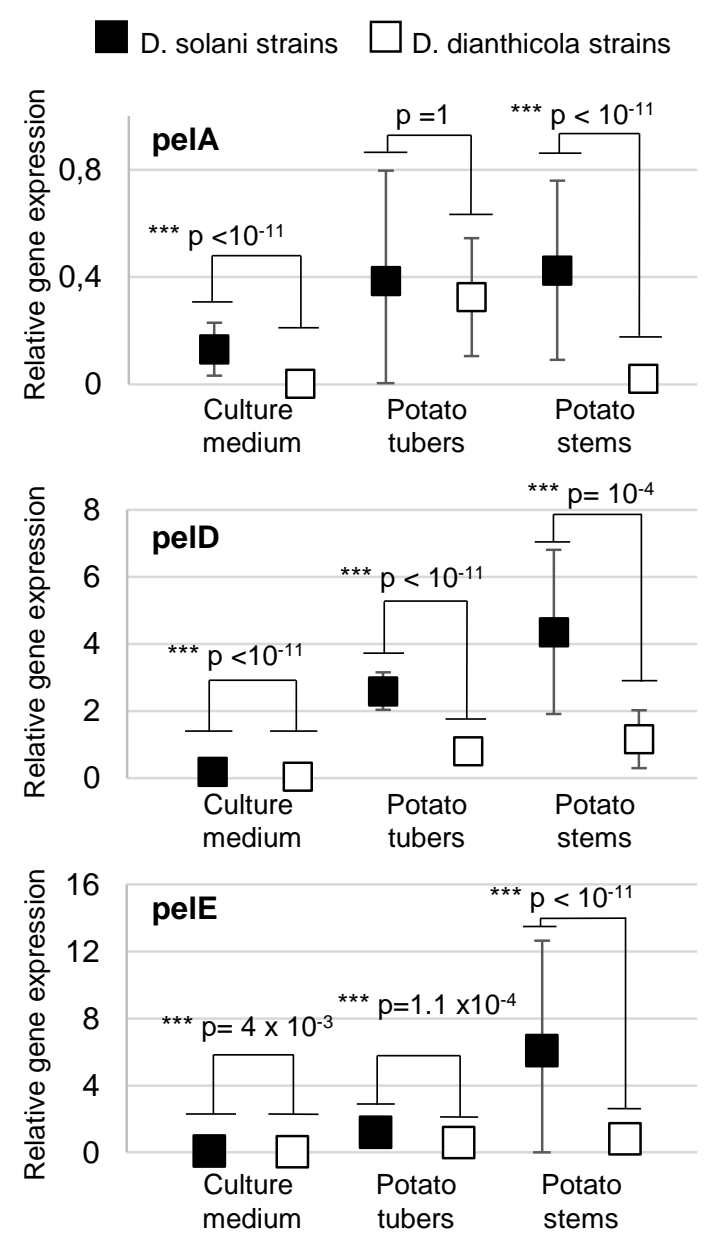

This article is protected by copyright. All rights reserved 
Figure 6

This article is protected by copyright. All rights reserved

(a) $\begin{array}{lr}490000 & 980000 \\ D . & \text { solani } 3337 \text { genome }(b p)\end{array}$

(b)

EU3296 S Ds0432.1 S$$
\text { (c) }
$$

$v f m B$

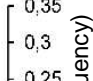

0.2 窇

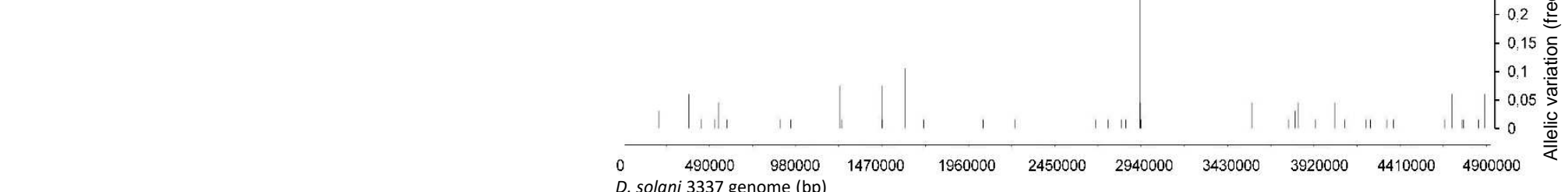

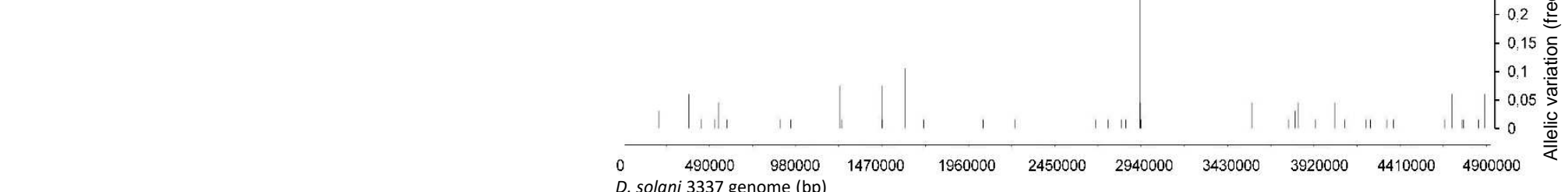
Me4a RNS13-37-1A
RNS14-33-1A
RNS10-58-3A Ka1a

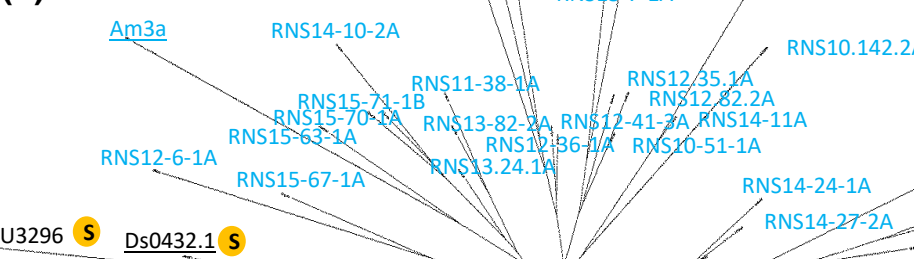

RNS10-TP258-34
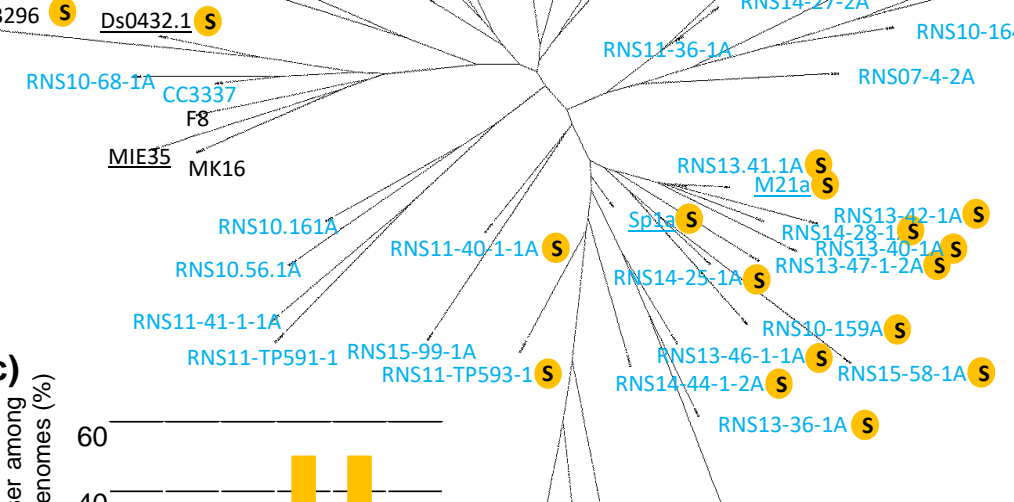

RNS13-35-1A S

RNS10-27-2A S

CC2276

CC2187 


\section{Figure 7}

(a)

(b)

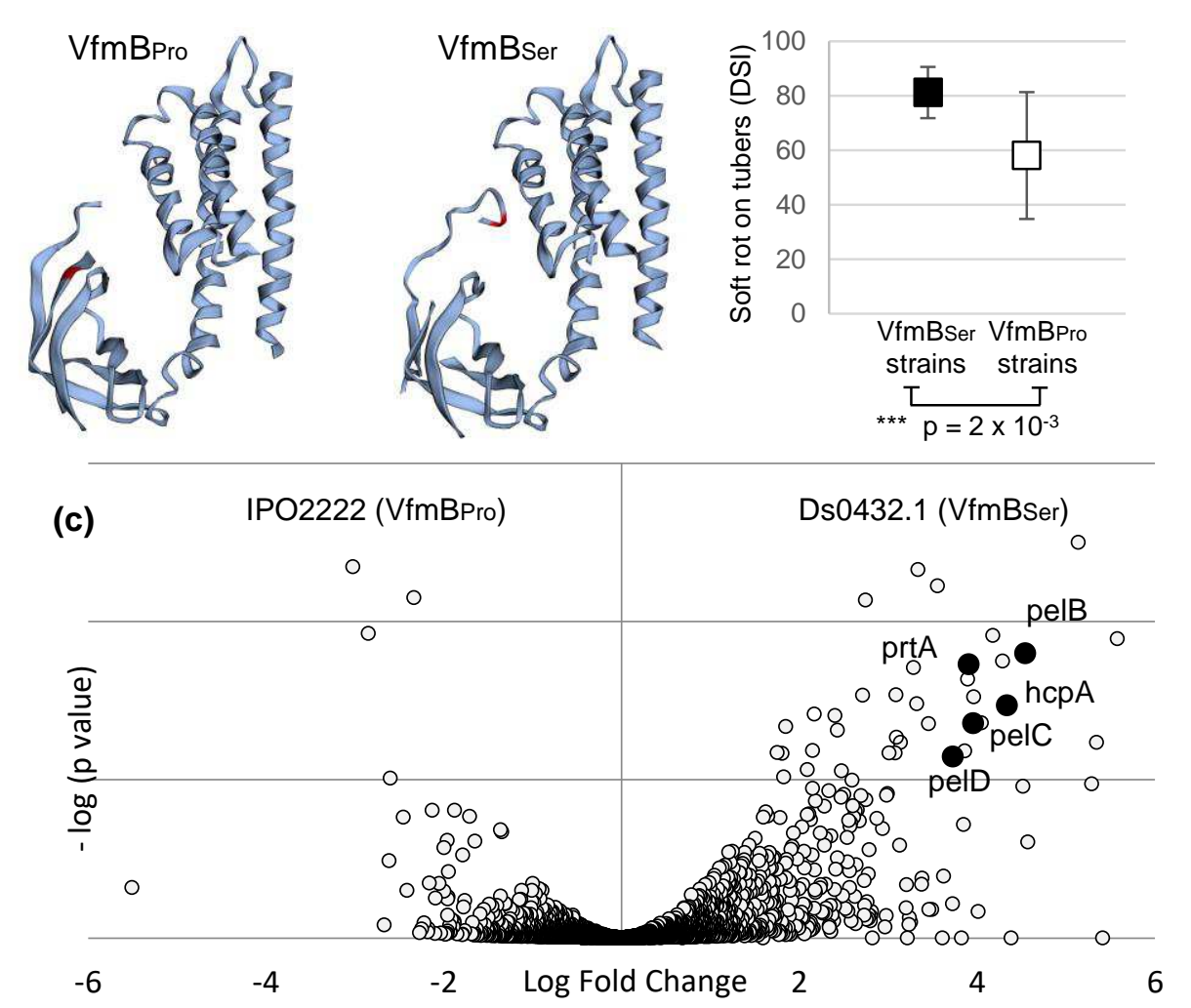

This article is protected by copyright. All rights reserved 


\section{Figure 8}

(a)

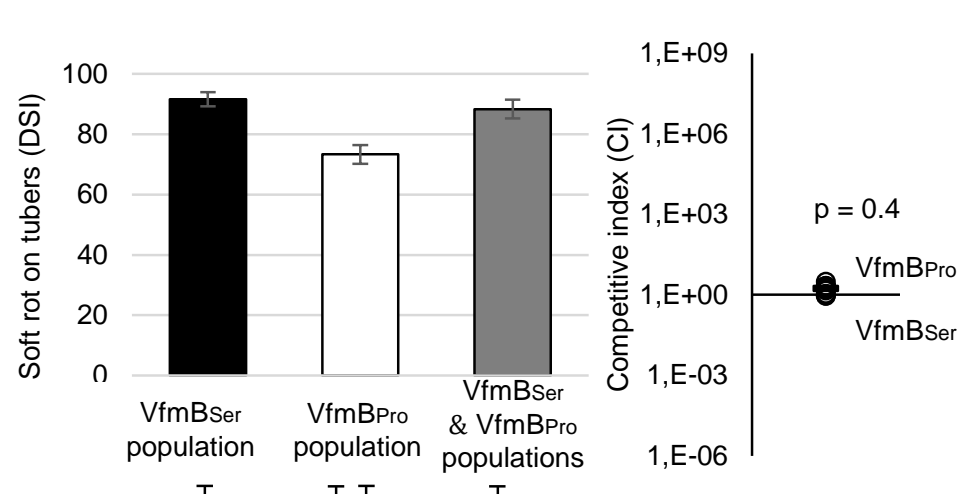

(b)
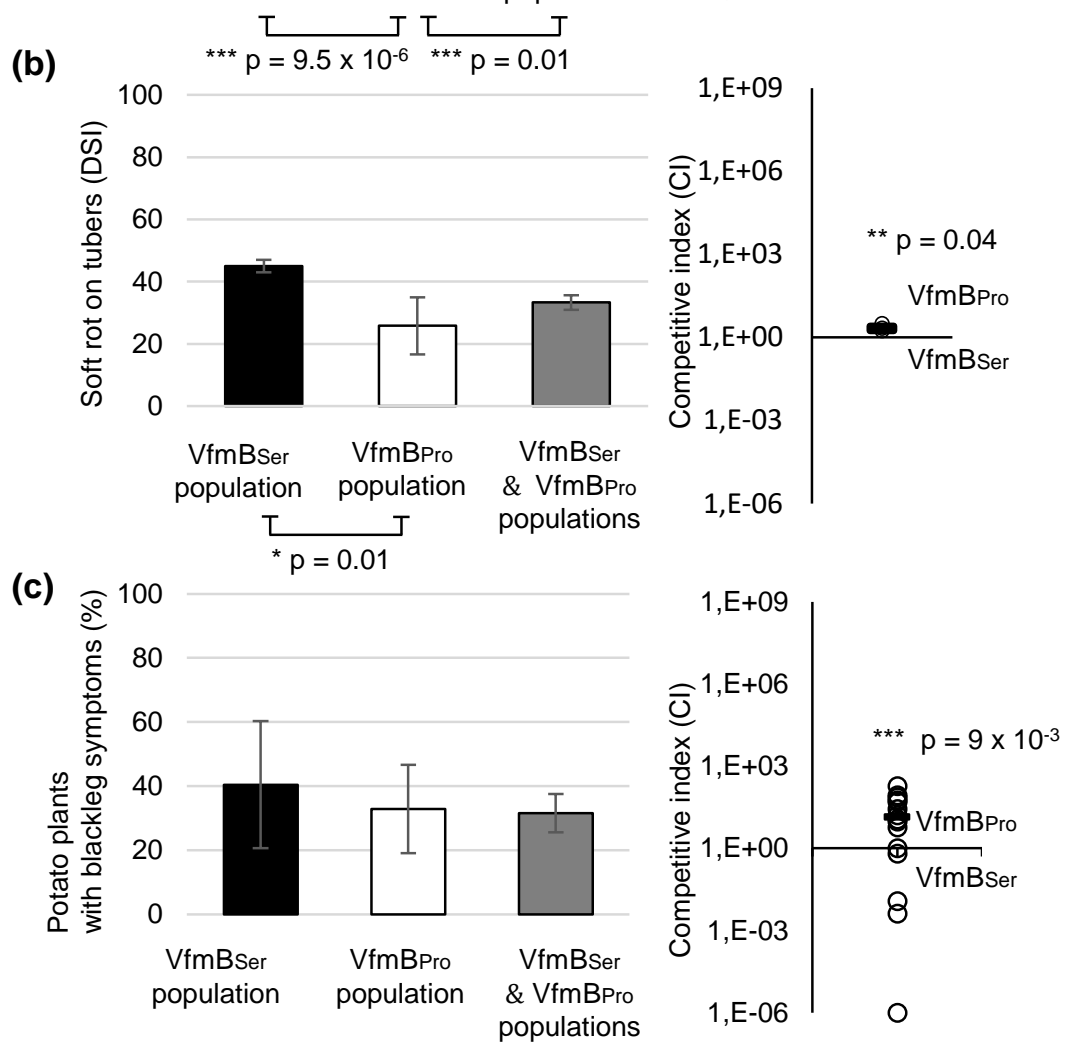

This article is protected by copyright. All rights reserved 